\title{
A LOGARITHMIC EPIPERIMETRIC INEQUALITY FOR THE OBSTACLE PROBLEM
}

\author{
MARIA COLOMBO, LUCA SPOLAOR, BOZHIDAR VELICHKOV
}

\begin{abstract}
For the general obstacle problem, we prove by direct methods an epiperimetric inequality at regular and singular points, thus answering a question of Weiss (Invent. Math., 138 (1999), 23-50). In particular at singular points we introduce a new tool, which we call logarithmic epiperimetric inequality, which yields an explicit logarithmic modulus of continuity on the $C^{1}$ regularity of the singular set, thus improving previous results of Caffarelli and Monneau [4, 2, 11.
\end{abstract}

Keywords: epiperimetric inequality, monotonicity formula, obstacle problem, free boundary, singular points

\section{INTRODUCTION}

In this paper we study the regularity of the free-boundary of nonnegative local minimizers $u$ of the functional

$$
\mathcal{E}(u):=\int|\nabla u|^{2} d x+\int \max \{u(x), 0\} d x .
$$

Our main result is a logarithmic epiperimetric inequality, which is a new tool for the study of the singular set of minimizers of variational energies. It is also an alternative approach to the regularity of the singular free boundary as proposed by Caffarelli [4, 2. Before we state it we recall that, given $u \in H^{1}\left(B_{1}\right)$, the Weiss' boundary adjusted energy of $u$ is defined by

$$
W(u)=\int_{B_{1}}|\nabla u|^{2} d x-2 \int_{\partial B_{1}} u^{2} d \mathcal{H}^{d-1}+\int_{B_{1}} \max \{u(x), 0\} d x .
$$

The class $\mathcal{K}$ of admissible blow-ups of $u$ at singular points is defined by

$$
\mathcal{K}:=\left\{Q_{A}: \mathbb{R}^{d} \rightarrow \mathbb{R}: Q_{A}(x)=x \cdot A x, A \text { symmetric non-negative with } \operatorname{tr} A=1 / 4\right\} .
$$

The energy $W$ is constant on $\mathcal{K}$, precisely we have $W\left(Q_{A}\right)=\frac{\omega_{d}}{8 d(d+2)}$, for every $Q_{A} \in \mathcal{K}$. We refer to this constant as to the energy density at the singular points and denote it by $\Theta$.

Theorem 1 (Logarithmic epiperimetric inequality at singular points). There are dimensional constants $\delta>0$ and $\varepsilon>0$ such that the following claim holds. For every non-negative function $c \in H^{1}\left(\partial B_{1}\right)$, with 2-homogeneous extension $z$ on $B_{1}$, satisfying

$$
\operatorname{dist}_{L^{2}\left(\partial B_{1}\right)}(c, \mathcal{K}) \leq \delta \quad \text { and } \quad 0 \leq W(z)-\Theta \leq 1,
$$

there is a non-negative function $h \in H^{1}\left(B_{1}\right)$ with $h=c$ on $\partial B_{1}$ satisfying the inequality

$$
W(h) \leq W(z)-\varepsilon(W(z)-\Theta)^{1+\gamma}, \quad \text { where } \quad\left\{\begin{array}{ll}
\gamma=0 & \text { if } d=2 \\
\gamma=\frac{d-1}{d+3} & \text { if } d \geq 3
\end{array} .\right.
$$

At flat points we recover the Weiss' epiperimetric inequality with a direct proof. To state it, recall that the collection $\mathcal{K}_{+}$of possible blow-ups at flat points is defined by

$$
\mathcal{K}_{+}:=\left\{q_{\nu}: \mathbb{R}^{d} \rightarrow \mathbb{R}: q_{\nu}(x)=(\max \{x \cdot \nu, 0\})^{2} \text { for some } \nu \in \mathbb{R}^{d} \text { such that }|\nu|=\frac{1}{2}\right\} .
$$

The energy $W$ is constant on $\mathcal{K}_{+}$, precisely we have $W\left(q_{\nu}\right)=\frac{\omega_{d}}{16 d(d+2)}$, for every $q_{\nu} \in \mathcal{K}_{+}$. We will refer to this constant as the energy density at the flat points and denote it by $\Theta_{+}$. 
Theorem 2 (Epiperimetric inequality at flat points). There are dimensional constants $\delta_{0}>0$, $\delta>0$ and $\varepsilon>0$ such that the following claim holds. For every non-negative function $c \in H^{1}\left(\partial B_{1}\right)$ satisfying

$$
\left\{x_{d}<-\delta_{0}\right\} \subset\{c=0\} \quad \text { and } \quad\left\|c-q_{e_{d}}\right\|_{L^{2}\left(\partial B_{1}\right)} \leq \delta
$$

there exists a non-negative function $h \in H^{1}\left(B_{1}\right)$ such that $h=c$ on $\partial B_{1}$ and

$$
W(h)-\Theta_{+} \leq(1-\varepsilon)\left(W(z)-\Theta_{+}\right),
$$

where $z$ is the 2-homogeneous extension of $c$ to $B_{1}$.

Theorem 2 was already proved by Weiss in [14] using a very elegant and innovative contradiction argument, later exploited also by Garofalo-Petrosyan-Garcia and Focardi-Spadaro in the context of the thin obstacle problem (see [9, 8]). However, the same proof works only at singular points of maximal and minimal dimension under some special assumptions on the projection of the trace on $\mathcal{K}$, which can be verified only in dimension $d=2$. Notice that the dimension of a singular point is the maximal $\operatorname{dim}(\operatorname{ker} A)$ among all $Q_{A} \in \mathcal{K}$ blow-ups of $u$ at the singular point. Hence, no epiperimetric inequality was known in the literature for the whole singular set, as it happens in Theorem 1 and Weiss himself suggests that "...it should however be possible to give a direct proof of the epiperimetric inequality which would then also cover singular sets of intermediate dimension" (see [14]). Theorems 1 and 2 answer affirmatively to this question, and in particular Theorem 1 is the first instance in the literature of an epiperimetric inequality of logarithmic type and the first instance in which the epiperimetric inequality for singular points has a direct proof. The methods developed to prove the epiperimetric inequality at singular points of any stratum have a quite general nature and will be applied to provide similar results in other problems, for instance in the case of the thin obstacle problem [5].

The proof of these theorems is direct (i.e. we produce explicit competitors) and it is remarkable in our opinion how the failure of Weiss' contradiction argument translates into a weakening of the epiperimetric inequality, that is the necessity of introducing the exponent $\gamma$ in (1.2). To explain this better, notice that, in analogy with Reifenberg and White's pioneering work (see [12, 16]) and similarly to previous work of the last two authors (see [13]), the key ingredients are

- a Fourier decomposition of the trace $c-q_{\nu}$ (resp. $c-Q_{A}$ ) onto the eigenfunctions of $\mathbb{S}^{d-1}$;

- an energy improvement with respect to $z$ obtained by taking the harmonic extension of the modes with homogeneity larger than two;

- a choice of $\nu$ (resp. A) to control the projection of $c-q_{\nu}$ (resp. $c-Q_{A}$ ) onto the eigenfunctions of homogeneity one and two, with the projection on the higher modes.

At flat points and at every point in dimension $d=2$, the estimate of the third bullet is linear, however in general dimension at singular points we can only prove a control of the form

$$
\left\|P\left(c-Q_{A}\right)\right\|_{H^{1}\left(\partial B_{1}\right)} \leq C\left\|(I d-P)\left(c-Q_{A}\right)\right\|_{H^{1}\left(\partial B_{1}\right)}^{1-\gamma} \quad \gamma \in(0,1),
$$

where $P$ denotes the projection on the modes relative to homogeneity two (see (4.6)). The reason for this different behavior is essentially the following: at the flat points we are able to eliminate the lower modes (the modes corresponding to homogeneity smaller than two) on a spherical cap by means of the choice of the vector $\nu$; this is possible since the space of admissible functions $q_{\nu}$ is an open manifold of the same dimension as the eigenspace corresponding to the lower modes, so we can apply an implicit function argument (see Lemma 3.2). At general singular points, we would like to eliminate the modes of homogeneity two, that is the modes corresponding to the eigenvalue $2 d$ on the sphere and whose eigenspace can be identified with the space of $d \times d$ real symmetric matrices $S_{d}(\mathbb{R})$. However, the positivity constraint on the competitor forces the choice of the matrix $A$ to be in the space of nonnegative symmetric matrices $S_{d}^{+}(\mathbb{R})$. Now these two spaces have the same dimension, but, due to the non-negativity assumption, the set $S_{d}^{+}(\mathbb{R}) \subset S_{d}(\mathbb{R})$ is not open, so we cannot apply the implicit function theorem here. Indeed, if we are in its interior, which corresponds to the singular points studied by Weiss, then the argument works and we can eliminate the second modes; but at the boundary of $S_{d}^{+}(\mathbb{R})$ an implicit function argument only provides us with a matrix in the larger space $S_{d}(\mathbb{R})$. This leaves us to estimate 
the difference between the element of $S_{d}(\mathbb{R})$, corresponding to the second modes of the trace $c$, and its projection on $S_{d}^{+}(\mathbb{R})$. We can do this by means of the additional condition that $c$ is positive, which suggests that this difference should be comparable to the higher modes of the trace, but because of capacitary reasons the bound comes with an exponent $\gamma \neq 0$. Roughly speaking, if the negative part produced by the second modes is very small, concentrated on a set of small capacity, then it can be compensated by a function with very small energy, much smaller than the distance to $S_{d}^{+}(\mathbb{R})$ in the space of symmetric matrices. In particular, it seems that this obstruction is of the same nature as the one that appears in [14], where the strong convergence of the traces cannot see the nodal sets of small capacity.

A similar phenomenon can be found in the theory of minimal surfaces. Indeed if we think about the collection of singular points of lower dimension as a minimal surface in codimension higher than one, then it is known the existence of non-integrable cones, that is cones with nonintegrable Jacobi fields. In this case the best possible rate of convergence to the blow-up is indeed logarithmic, as shown in [1].

We should remark that an estimate of the form (1.2) is essentially the best one can get by using only the positivity of the trace $c$ (see Example 1). It follows that Theorem 1 is essentially optimal, as it concerns positive traces on the sphere. However it is conceivable that for solutions of the obstacle problem a better inequality could be obtained, by using more properties of the minimizers.

It is well known that Theorem 2 leads to the uniqueness of the blow-up at every flat point and also to the $C^{1, \alpha}$ regularity of the regular part of the free-boundary (see [14]). We show that Theorem 1 yields the uniqueness of the blow-up and the $C^{1}$ regularity of the singular set, with an explicit logarithmic modulus of continuity. This is an improvement on the results of Caffarelli and Monneau, where such a modulus arises by contradiction arguments and is therefore not explicit (see [4, 11]). The method of the present paper is flexible enough to cover more general and nonlinear functionals, such as the area. The stratification of the singular set for the area functional, even in the context of Riemannian manifolds, and the $C^{1}$ regularity of the strata were recently obtained in [7, 6]. Before giving the precise statements, we need some additional definitions. We split the free-boundary of a minimizer $u$ in regular and singular part, defined as

$$
\operatorname{Reg}(u):=\left\{x \in \partial\{u>0\} \cap \Omega \text { : any blow up at } x \text { is of the form } q_{\nu} \in \mathcal{K}_{+}\right\}
$$

$$
\operatorname{Sing}(u):=\left\{x \in \partial\{u>0\} \cap \Omega \text { : at least one blow up at } x \text { is not of the form } q_{\nu} \in \mathcal{K}_{+}\right\}
$$

Their regularity is the content of the following results.

Theorem 3 (Uniqueness of the blow up and logarithmic convergence). Let $\gamma=\frac{d-1}{d+3}, \Omega \subset \mathbb{R}^{d}$ be an open set and $u \in H^{1}(\Omega)$ a minimizer of $\mathcal{E}$. Then the blow up of $u$ at each point of the free boundary $\partial\{u>0\} \cap \Omega$ is unique. Moreover, the following convergence holds.

(1) For every $x_{0} \in \operatorname{Reg}(u) \cap \Omega$ there exist $r:=r\left(x_{0}\right), C:=C\left(x_{0}\right)$ and $\nu\left(x_{0}\right) \in \mathbb{R}^{d}$, with $\left|\nu\left(x_{0}\right)\right|=1 / 2$, such that

$$
\int_{\partial B_{1}}\left|u_{x_{1}, r}-q_{\nu\left(x_{1}\right)}\right| d \mathcal{H}^{n-1} \leq C r^{\frac{(n+2) \varepsilon}{2(1-\varepsilon)}}, \quad \text { for every } \quad r \leq r_{0} \text { and } x_{1} \in \operatorname{Reg}(u) \cap B_{r}\left(x_{0}\right) .
$$

(2) For every $x_{0} \in \operatorname{Sing}(u)$, there exist $r:=r\left(x_{0}\right), C:=C\left(x_{0}\right)$ and $Q_{x_{0}} \in \mathcal{K}$ such that

$$
\int_{\partial B_{1}}\left|u_{x_{0}, r}-Q_{x_{0}}\right| d \mathcal{H}^{n-1} \leq C(\log r)^{-\frac{1-\gamma}{2 \gamma}}, \quad \text { for every } \quad r \leq r_{0}
$$

The next regularity result recovers all the previously known results and improves the regularity of the singular set to $C^{1, \log }$. Before stating it we need to make precise what we mean by singular points of intermediate dimension. Given $k=0, \ldots, d-1$, we define the singular set of dimension 
$k$ (also called $k$-th stratum) $S_{k}(u)$ as

$$
\begin{aligned}
S_{k}(u) & :=\left\{x \in \operatorname{Sing}(u): \operatorname{dim}(\operatorname{ker}(A)) \leq k \text { for every blow-up } Q_{A} \in \mathcal{K} \text { of } u \text { at } x\right\} \\
& =\bigcup_{l=1}^{k}\left\{x \in \operatorname{Sing}(u): \operatorname{dim}(\operatorname{ker}(A))=l \text { for the unique blow-up } Q_{A} \in \mathcal{K} \text { of } u \text { at } x\right\},
\end{aligned}
$$

where the equivalence of the two definitions is guaranteed by Theorem 3, In the case of the stratum $S_{0}(u)$ the inequality (1.5) can be improved to $C^{1, \beta}$ convergence.

Theorem 4 (Regularity of the free boundary). Let $\varepsilon>0$ be the constant from Theorem 2 , $\beta=\frac{(d+2) \varepsilon}{2(1-\varepsilon)}\left(1+\frac{(d+2) \varepsilon}{2(1-\varepsilon)}\right)^{-1}, \Omega \subset \mathbb{R}^{d}$ be an open set and $u \in H^{1}(\Omega)$ a minimizer of $\mathcal{E}$. Then

(1) $\operatorname{Reg}(u)$ is locally the graph of a $C^{1, \beta}$ function; namely, for every $x_{0} \in \operatorname{Reg}(u) \cap \Omega$ there exists $r:=r\left(x_{0}\right)$ such that $\operatorname{Reg}(u) \cap B_{r}\left(x_{0}\right)$ is a $C^{1, \beta}$ - submanifold of dimension $(d-1)$;

(2) For every $k=0, \ldots, d-1, S_{k}(u)$ is contained in the union of countably many submanifolds of dimension $k$ and class $C^{1, l o g}$; namely for every $x_{0} \in \operatorname{Sing}(u) \cap \Omega$ there exists $r_{0}:=r_{0}\left(x_{0}\right)$ and $C:=C\left(x_{0}\right)$ such that a logarithmic estimate holds

$$
\left|Q\left(x_{1}\right)-Q\left(x_{2}\right)\right| \leq C\left(\log \left|x_{1}-x_{2}\right|\right)^{-\frac{1-\gamma}{2 \gamma}} \quad \text { for any } x_{1}, x_{2} \in S_{k} \cap B_{r}\left(x_{0}\right) .
$$

(3) If the dimension $d=2$, then we have the estimate

$$
\left|Q\left(x_{1}\right)-Q\left(x_{2}\right)\right| \leq C\left|x_{1}-x_{2}\right|^{\beta} \quad \text { for any } x_{1}, x_{2} \in S_{k} \cap B_{r}\left(x_{0}\right),
$$

for $k=1,2$, where $\beta$ is the same as in (1). In particular $S_{0}$ consists of isolated points and $S_{1}$ is contained in the union of at most countably many curves of class $C^{1, \beta}$.

Remark 1.1. Thanks to a result of Caffarelli and Riviére (see [3]) it is possible to improve (3) to the following result: the boundary of a connected component of the interior of the free-boundary is analytic except at finitely many singular points.

Theorems 3 and 4 remain true if we consider a Hölder continuous weight function $q: \Omega \rightarrow \mathbb{R}^{+}$ and more general functionals, for instance

$$
\mathcal{E}_{q}(u):=\int_{\Omega}\left[|\nabla u|^{2}+q(x)|u|\right] d x, \quad \mathcal{A}_{q}(u):=\int_{\Omega}\left[\sqrt{|\nabla u|^{2}+1}+q(x)|u|\right] d x .
$$

In this case, the regular and singular parts at a given point $x$ are defined as for $\mathcal{E}$, up to a constant which depends on $q(x)$. Given $u \in H^{1}\left(B_{1}\right)$ positive minimizer of $\mathcal{E}$, we define

$$
\operatorname{Reg}_{q}(u):=\left\{x \in \partial\{u>0\} \cap \Omega: \text { any blow up at } x \text { is of the form } q_{\nu} \text { for }|\nu|=\frac{q(x)}{2}\right\},
$$

$\operatorname{Sing}_{q}(u):=\left\{x \in \partial\{u>0\} \cap \Omega\right.$ : at least one blow up at $x$ is not of the form $q_{\nu}$, for $\left.|\nu|=\frac{q(x)}{2}\right\}$, $S_{q, k}(u):=\left\{x \in \operatorname{Sing}_{q}(u): \operatorname{dim}(\operatorname{ker}(A)) \leq k\right.$ for every blow-up $Q_{A} \in \mathcal{K}$ of $u$ at $\left.x\right\}$.

Theorem 5 (Hölder continuous weight functions and area functional). Let $\alpha>0, \Omega \subset \mathbb{R}^{d}$ be an open set and $q \in C^{0, \alpha}\left(\Omega ; \mathbb{R}^{+}\right)$be an Hölder continuous function such that $q \geq c_{q}>0$, where $c_{q}$ is a given constant. Let $u \in H^{1}(\Omega)$ be a minimizer of $\mathcal{E}_{q}$ or $\mathcal{A}_{q}$. Then the blow up of $u$ at each point of the free boundary $\partial\{u>0\} \cap \Omega$ is unique and

(1) there exists $\beta>0$ such that $\operatorname{Reg}_{q}(u)$ is locally the graph of a $C^{1, \beta}$ function;

(2) For every $k=0, \ldots, d-1, S_{q, k}(u)$ is contained in the union of countably many submanifolds of dimension $k$ and class $C^{1, l o g}$; namely for every $x_{0} \in \operatorname{Sing}_{q}(u) \cap \Omega$ there exists $r_{0}:=$ $r_{0}\left(x_{0}\right)$ and $C:=C\left(x_{0}\right)$ such that a logarithmic estimate holds

$$
\left|Q\left(x_{1}\right)-Q\left(x_{2}\right)\right| \leq C\left(\log \left|x_{1}-x_{2}\right|\right)^{-\frac{1-\gamma}{2 \gamma}} \quad \text { for any } x_{1}, x_{2} \in \operatorname{Sing}_{q}(u) \cap B_{r}\left(x_{0}\right) .
$$

Compared to a similar result obtained from the epiperimetric inequality with indirect proof, here we have quantitative estimates as (1.8) and, for the regular set, an explicit Hölder regularity in terms of the dimension and the Hölder exponent of $q$. 
Organization of the paper. The paper is divided in four short sections. In Section 2 we fix notations and easy preliminary computations. In Section 3 we prove the Weiss epiperimetric inequality Theorem 2, while Section 4 is dedicated to Theorem 1 . Finally, in Section 5 we apply these two theorems to deduce the various regularity results.

\section{Preliminaries}

In this section we fix some notations and we recall some known facts about the solutions of the obstacle problem, their blow-up limits, the decomposition of the free boundary in a regular and singular part and its realtion with the Weiss boundary adjusted functional. The final subsection is dedicated to the Fourier analysis on the unit sphere in $\mathbb{R}^{d}$, which will be useful for both Theorems 1 and 2 ,

2.1. Notations. We will use the following notations. $B_{1}$ is the $d$-dimensional unit ball centered in zero and $\omega_{d}=\left|B_{1}\right|$ is the Lebesgue measure of $B_{1}$. We denote by $\mathbb{S}^{d-1}$ or $\partial B_{1}$ the unit $(d-1)$-dimensional sphere in $\mathbb{R}^{d}$ equipped with the $(d-1)$-dimensional Hausdorff measure $\mathcal{H}^{d-1}$. $\theta$ will denote the variable on the sphere $\partial B_{1}$. For an open set $\Omega$ in $\mathbb{R}^{d}$ or on the sphere $\mathbb{S}^{d-1}$ we will denote by $H^{1}(\Omega) \subset L^{2}(\Omega)$ the Sobolev space of weakly differentiable functions on $\Omega$ with gradients in $L^{2}\left(\Omega ; \mathbb{R}^{d}\right)$ and by $H_{0}^{1}(\Omega)$ the space of functions $H^{1}(\Omega)$ which are zero on $\partial \Omega$.

For a function $f: \mathbb{R}^{d} \rightarrow \mathbb{R}$ we denote by $f_{+}$its positive part, $f_{+}(x)=\max \{f(x), 0\}$. For instance, given a vector $\nu \in \mathbb{R}^{d}$ we will often use the notations

$$
(x \cdot \nu)_{+}=\max \{x \cdot \nu, 0\} \quad \text { and } \quad(x \cdot \nu)_{+}^{2}=(\max \{x \cdot \nu, 0\})^{2},
$$

where $x \cdot \nu$ is the scalar product of the vectors $x$ and $\nu$ in $\mathbb{R}^{d}$.

2.2. Weiss boundary adjusted energy. For a function $u \in H^{1}(\Omega)$, with $\Omega \subset \mathbb{R}^{d}$, we denote by $W, W_{0}$ and $\widetilde{W}$ the functionals

$$
\begin{gathered}
W_{0}\left(u, x_{0}, r\right):=\frac{1}{r^{d+2}} \int_{B_{r}\left(x_{0}\right)}|\nabla u|^{2} d x-\frac{2}{r^{d+3}} \int_{\partial B_{r}\left(x_{0}\right)} u^{2} d \mathcal{H}^{d-1}, \\
\widetilde{W}\left(u, x_{0}, r\right):=W_{0}\left(u, x_{0}, r\right)+\frac{1}{r^{d+2}} \int_{B_{r}\left(x_{0}\right)} u(x) d x, \\
W\left(u, x_{0}, r\right):=W_{0}\left(u, x_{0}, r\right)+\frac{1}{r^{d+2}} \int_{B_{r}\left(x_{0}\right)} \max \{u(x), 0\} d x,
\end{gathered}
$$

where $x_{0} \in \partial\{u>0\}$ and $0<r<\operatorname{dist}\left(x_{0}, \partial \Omega\right)$, and we notice that for non-negative functions $u \in H^{1}\left(B_{1}\right)$ we have $\widetilde{W}\left(u, x_{0}, r\right)=W\left(u, x_{0}, r\right)$. In particular, we set

$$
W(u, 0, r)=W(u, r) \quad \text { and } \quad W(u, 1)=W(u),
$$

and we recall the scaling property

$$
W\left(u, x_{0}, r\right)=W\left(u_{r, x_{0}}\right), \quad \text { where } \quad u_{r, x_{0}}(x)=\frac{u\left(r x+x_{0}\right)}{r^{2}} .
$$

For any $u \in H^{1}(\Omega)$ the following identity holds for $x_{0} \in \partial\{u>0\}$ and $0<r<\operatorname{dist}\left(x_{0}, \partial \Omega\right)$

$$
\frac{d}{d r} W\left(u, x_{0}, r\right)=\frac{d+2}{r}\left[W\left(z_{r, x_{0}}, 1\right)-W\left(u_{r, x_{0}}, 1\right)\right]+\frac{1}{r} \int_{\partial B_{1}}\left|x \cdot \nabla u_{r, x_{0}}-2 u_{r, x_{0}}\right|^{2} d \mathcal{H}^{d-1}
$$

where $z_{r, x_{0}}(x):=|x|^{2} u_{r, x_{0}}\left(\frac{x}{|x|}\right)$ (see for instance [14]). 
2.3. Global homogeneous solutions of the obstacle problem. Wa say that the function $u_{0}: \mathbb{R}^{d} \rightarrow \mathbb{R}$ is a blow-up limit of $u$ in the point $x_{0}$, if

$$
u_{0}=\lim _{n \rightarrow \infty} u_{r_{n}, x_{0}} \quad \text { for some sequence }\left(r_{n}\right)_{n} \text { with } \lim _{n \rightarrow \infty} r_{n}=0,
$$

where the converegnce is locally uniform and strong in $H_{l o c}^{1}\left(\mathbb{R}^{d}\right)$. Thanks to work of Caffarelli (see [2]), it is well known that $u_{0}$ is a global homogeneous solution of the obstacle problem. Precisely, $u_{0} \in \mathcal{K} \cup \mathcal{K}_{+}($introduced in (1.1) and (1.3) $)$. Moreover, we claim that

$W(Q)=\frac{\omega_{d}}{8 d(d+2)}=: \Theta$ for every $Q \in \mathcal{K}, \quad$ and $\quad W(q)=\frac{\omega_{d}}{16 d(d+2)}=: \Theta_{+}$for every $q \in \mathcal{K}_{+}$.

Indeed, for every $Q_{A} \in \mathcal{K}$ we have $\Delta Q_{A}=2 \operatorname{tr} A=\frac{1}{2}$ and so an integration by parts gives

$$
W_{0}\left(Q_{A}\right)=\int_{B_{1}}\left|\nabla Q_{A}\right|^{2}-2 \int_{\partial B_{1}} Q_{A}^{2}=-\int_{B_{1}} Q_{A} \Delta Q_{A}=-\frac{1}{2} \int_{B_{1}} Q_{A} .
$$

Since $Q_{A}$ is positive and denoting $\left(a_{i j}\right)_{i j}$ the coefficients of the matrix $A$, we get

$$
W\left(Q_{A}\right)=\widetilde{W}\left(Q_{A}\right)=W_{0}\left(Q_{A}\right)+\int_{B_{1}} Q_{A}=\frac{1}{2} \int_{B_{1}} Q_{A}=\frac{1}{2} \int_{B_{1}} \sum_{i=1}^{d} a_{i i} x_{i}^{2} d x=\frac{\operatorname{tr} A}{2} \int_{B_{1}} x_{d}^{2} d x=\Theta .
$$

Analogously, for any $q_{\nu} \in \mathcal{K}_{+}$we have $\Delta q_{\nu}=2|\nu|^{2}=\frac{1}{2}$ on the set $\{x \cdot \nu>0\}$, so that

$$
W\left(q_{\nu}\right)=\widetilde{W}\left(q_{\nu}\right)=W_{0}\left(q_{\nu}\right)+\int_{B_{1}} q_{\nu}=\frac{1}{2} \int_{B_{1}} q_{\nu}=\frac{|\nu|^{2}}{2} \int_{B_{1} \cap\left\{x_{d}>0\right\}} x_{d}^{2} d x=\frac{\omega_{d}}{16 d(d+2)}=\Theta_{+} .
$$

2.4. Regular and singular free boundaries. We recall that, as observed by Weiss [15], a consequence of (2.1) is that if $u \in H^{1}(\Omega)$ is a nonnegative minimizer of $\mathcal{E}$ in the open set $\Omega \subset \mathbb{R}^{d}$ and $x_{0} \in \Omega$, then the function $r \mapsto W\left(u, x_{0}, r\right)$ is nondecreasing (in its domain of definition $\left.0<r<\operatorname{dist}\left(x_{0}, \partial \Omega\right)\right)$ and there exists the limit

$$
\Theta_{u}\left(x_{0}\right):=\lim _{r \rightarrow 0} W\left(u, x_{0}, r\right)=\inf _{r>0} W\left(u, x_{0}, r\right)=\lim _{r \rightarrow 0} W\left(u_{r, x_{0}}\right) .
$$

Moreover, if $q$ is a blow-up limit of the minimizer $u$ in $x_{0}$, then

$$
W(q)=\lim _{n \rightarrow \infty} W\left(u_{r_{n}, x_{0}}\right)=\Theta_{u}\left(x_{0}\right) .
$$

Since we have that $q \in \mathcal{K} \cup \mathcal{K}_{+}$, there are only two possible values for the energy density $\Theta_{u}\left(x_{0}\right)$ :

$$
\Theta_{u}\left(x_{0}\right)=\Theta_{+} \quad \text { or } \quad \Theta_{u}\left(x_{0}\right)=\Theta .
$$

Hence we can redefine the regular and the singular part of the free boundary $\partial\{u>0\} \cap \Omega$ as

$$
\begin{aligned}
& \operatorname{Reg}(u)=\left\{x \in \partial\{u>0\} \cap \Omega: \Theta_{u}(x)=\Theta_{+}\right\}, \\
& \operatorname{Sing}(u)=\left\{x \in \partial\{u>0\} \cap \Omega: \Theta_{u}(x)=\Theta\right\} .
\end{aligned}
$$

By definition the free boundary $\partial\{u>0\} \cap \Omega$ is a disjoint union of $\operatorname{Reg}(u)$ and $\operatorname{Sing}(u)$. Moreover, by the definition of the density (2.3) and the fact that $x_{0} \mapsto W\left(u, x_{0}, r\right)$ is continuous, the function $x_{0} \mapsto \Theta_{u}\left(x_{0}\right)$ is upper semicontinuous. This, together with (2.2) and the fact that all the blow-up limits are in $\mathcal{K} \cup \mathcal{K}_{+}$, finally gives the following characterization of $\operatorname{Reg}(u)$ and $\operatorname{Sing}(u)$ :

- the set $\operatorname{Reg}(u)$ is a relatively open subset of the free boundary $\partial\{u>0\}$, and every blow-up limit at a point of $\operatorname{Reg}(u)$ is of the form $q_{\nu}$, for some $q_{\nu} \in \mathcal{K}_{+}$;

- the set $\operatorname{Sing}(u)$ is closed, and every blow-up limit at a point of $\operatorname{Sing}(u)$ is of the form $Q_{A}$, for some $Q_{A} \in \mathcal{K}$. 
2.5. Eigenvalues and eigenfunctions on subdomains of the sphere. Let $S \subseteq \mathbb{S}^{d-1}$ be an open set. Let $0<\lambda_{1} \leq \lambda_{2} \leq \cdots \leq \lambda_{j} \leq \ldots$ be the eigenvalues (counted with multiplicity) of the spherical Laplace-Beltrami operator with Dirichlet conditions on $\partial S$ and $\left\{\phi_{j}\right\}_{j \geq 1}$ be the corresponding eigenfunctions, that is the solutions of the problem

$$
-\Delta_{\mathbb{S}^{d-1}} \phi_{j}=\lambda_{j} \phi_{j} \quad \text { in } \quad S, \quad \phi_{j}=0 \quad \text { on } \quad \partial S, \quad \int_{S} \phi_{j}^{2}(\theta) d \mathcal{H}^{d-1}(\theta)=1 .
$$

Any function $\psi \in H_{0}^{1}(S)$ can be decomposed as $\psi(\theta)=\sum_{j=1}^{\infty} c_{j} \phi_{j}(\theta)$. The following lemma compares the energies of 2-homogeneous and $\alpha$-homogeneous functions by means of the Fourier decomposition of their common values on $\partial B_{1}$.

Lemma 2.1. Let $\psi \in H_{0}^{1}(S)$ and consider the 2-homogeneous extension $\varphi(r, \theta)=r^{2} \psi(\theta)$ and the $\alpha$-homogeneous extension $\tilde{\varphi}(r, \theta)=r^{\alpha} \psi(\theta)$ respectively of $\psi$ to $B_{1}$, for some $\alpha>2$. Set

$$
\varepsilon_{\alpha}:=\frac{\alpha-2}{d+\alpha} \quad \text { and } \quad \lambda_{\alpha}:=\alpha(\alpha+d-2) .
$$

Then the following inequality holds

$$
W_{0}(\tilde{\varphi})-\left(1-\varepsilon_{\alpha}\right) W_{0}(\varphi)=\frac{\varepsilon_{\alpha}}{d+2 \alpha-2} \sum_{j=1}^{\infty}\left(-\lambda_{j}+\lambda_{\alpha}\right) c_{j}^{2} .
$$

Proof. Since $\left\|\varphi_{j}\right\|_{L^{2}\left(\partial B_{1}\right)}=1$ and $\left\|\nabla_{\theta} \varphi_{j}\right\|_{L^{2}\left(\partial B_{1}\right)}=\lambda_{j}$ for every $j \in\{0\} \cup \mathbb{N}$, the energy of the $\alpha$-homogeneous function $\tilde{\varphi}(r, \theta)=r^{\alpha} \psi(\theta)$ can be written as

$$
\begin{aligned}
W_{0}(\tilde{\varphi}) & =\sum_{j=1}^{\infty} c_{j}^{2}\left(\int_{0}^{1} r^{d-1} d r \int_{S} d \mathcal{H}^{d-1}\left[\alpha^{2} r^{2 \alpha-2} \phi_{j}^{2}+r^{2 \alpha-2}\left|\nabla_{\theta} \phi_{j}\right|^{2}\right]-2 \int_{S} \phi_{j}^{2} d \mathcal{H}^{d-1}\right) \\
& =\sum_{j=1}^{\infty} c_{j}^{2}\left(\frac{\alpha^{2}+\lambda_{j}}{d+2 \alpha-2}-2\right) .
\end{aligned}
$$

When $\alpha=2$ and $\varphi(r, \theta)=r^{2} \psi(\theta)$, we get

$$
W_{0}(\varphi)=\sum_{j=1}^{\infty} c_{j}^{2}\left(\frac{4+\lambda_{j}}{d+2}-2\right) .
$$

We now notice that for every $\lambda$ we have

$$
\left(\frac{\alpha^{2}+\lambda}{d+2 \alpha-2}-2\right)-\left(1-\varepsilon_{\alpha}\right)\left(\frac{4+\lambda}{d+2}-2\right)=\frac{\lambda(2-\alpha)}{(d+\alpha)(d+2 \alpha-2)}+\frac{(\alpha-2) \alpha(\alpha+d-2)}{(d+\alpha)(d+2 \alpha-2)},
$$

which concludes the proof of Lemma 2.1,

The above lemma, in particular, shows that if the decomosition of $\psi$ involves only eigenfunctions corresponding to eigenvalues $\lambda_{j} \geq \lambda_{\alpha}$, then the $\alpha$-homogeneous extension $\tilde{\varphi}$ has a strictly lower energy than the two-homogeneous extension $\varphi$. In order to choose appropriately $\alpha$ we will need some additional information on the spectrum of the Laplacian on $S$. We recall that the function $\phi_{j}: S \rightarrow \mathbb{R}$ is a solution of the first equation in (2.4) if and only if its $\alpha_{j}$-homogeneous extension $\varphi_{j}(r, \theta)=r^{\alpha_{j}} \phi_{j}(\theta)$ is harmonic in the cone $\left\{(r, \theta) \in \mathbb{R}^{+} \times \partial B_{1}: \theta \in S\right\}$, where the homogeneity $\alpha_{j}$ is uniquely determined by the identity $\lambda_{j}=\alpha_{j}\left(\alpha_{j}+d-2\right)$.

The spectrum on the sphere $\mathbb{S}^{d-1}$. By the fact that the homogeneous harmonic functions in $\mathbb{R}^{d}$ are necessarily polynomials, we have that:

- $\lambda_{1}=0$ and the corresponding eigenfunction is the constant $\phi_{1}=\left|\partial B_{1}\right|^{-1 / 2}=\left(d \omega_{d}\right)^{-1 / 2}$.

- $\lambda_{2}=\cdots=\lambda_{d+1}=d-1$, the corresponding homogeneity constants are $\alpha_{2}=\cdots=\alpha_{d+1}=$ 1 and the corresponding eigenspace coincides with the space of linear functions in $\mathbb{R}^{d}$. 
- $\lambda_{d+2}=\cdots=\lambda_{d(d+3) / 2}=2 d$, the corresponding homogeneity constants are $\alpha_{d+2}=\cdots=$ $\alpha_{d(d+3) / 2}=2$. The corresponding eigenspace has dimension $d(d-1) / 2$ and is generated by the (restrictions to $\mathbb{S}^{d-1}$ of the) two-homogeneous harmonic polynomials:

$$
E_{2 d}=\left\{Q_{A}: \mathbb{R}^{d} \rightarrow \mathbb{R}: Q_{A}(x)=x \cdot A x, A \text { symmetric with } \operatorname{tr} A=0\right\} .
$$

- If $j>d(d+3) / 2$ (that is $\left.\lambda_{j}>2 d\right)$, then $\lambda_{j} \geq 3(3+d-2)=3(d+1)$.

The spectrum on the half-sphere $\partial B_{1}^{+}=\left\{x_{d}>0\right\} \cap \partial B_{1}$. We notice that the odd extension (with respect to the plane $\left\{x_{d}=0\right\}$ ) of any eigenfunction $\phi_{j}$ on the half-sphere $\partial B_{1}^{+}$is an eigenfunction on the entire sphere $\partial B_{1}$, which is zero on the equator $\left\{x_{d}=0\right\} \cap \partial B_{1}$. Thus, one can easily deduce that:

- $\lambda_{1}=d-1$ and the corresponding eigenfunction is $\phi_{1}(x)=\frac{x_{d}}{\sqrt{\omega_{d}}}$.

- $\lambda_{2}=\cdots=\lambda_{d}=2 d$, the corresponding homogeneity constants are $\alpha_{2}=\cdots=\alpha_{d}=2$ and the corresponding eigenspace $E_{2 d}$ has dimension $(d-1)$ and is generated by the polynomials

$$
Q_{j}(x)=x_{d} x_{j-1}, \quad \text { for every } \quad j=2, \ldots, d .
$$

- If $j>d$ (that is $\lambda_{j}>2 d$ ), then $\lambda_{j} \geq 3(3+d-2)=3(d+1)$.

The spectrum on the spherical cap $S_{\delta}=\partial B_{1} \cap\left\{x_{d}>-\delta\right\}$. We first notice that the spectrum $\left\{\lambda_{j}(\delta)\right\}_{j \geq 1}$ of the spherical cap $S_{\delta}$ varies continuously with respect to $\delta$. Thus, for $\delta>0$ small enough (smaller than some dimensional constant), we have

- $\lambda_{1}(\delta)$ is simple (isolated) eigenvalue and $\lambda_{1}(\delta) \leq d-1$;

- $d-1<\lambda_{j}(\delta)<2 d$, for every $j=2, \ldots, d$;

- $\lambda_{j}(\delta) \geq 3 d$, for every $j>d$.

Moreover, a standard separation of variables argument gives that:

- the first eigenfunction $\phi_{1}$ on $S_{\delta}$ is positive and depends only on the first variable $x_{d}$, that is $\phi_{1}(x)=\phi_{1}\left(x_{d}\right)$.

- the eigenfunctions $\phi_{2}, \ldots, \phi_{d}$ correspond to the same eigenvalue $\lambda_{2}(\delta)=\cdots=\lambda_{d}(\delta)$ and there is a function $\phi=\phi\left(x_{d}\right)$ such that

$$
\phi_{j}(x)=x_{j-1} \phi\left(x_{d}\right) \quad \text { for every } \quad j=2, \ldots, d .
$$

\section{The ePiPerimetric inequality at flat Points: Proof of Theorem 2}

In order to prove Theorem 2 we decompose the function $z$ as

$$
z=q_{\nu}+\varphi
$$

where $q_{\nu}(x)=(x \cdot \nu)_{+}^{2}$ and $\nu \in \mathbb{R}^{d}$ to be chosen later. We then replace the 2-homogeneous function $\varphi(r, \theta)=r^{2} \phi(\theta)$ by an $\alpha$-homogeneous function $\tilde{\varphi}(r, \theta)=r^{\alpha} \phi(\theta)$, for some $\alpha>2$. The final competitor will be of the form

$$
h=q_{\nu}+\tilde{\varphi}
$$

and $\varepsilon=\varepsilon_{\alpha}$ will be given by

$$
\varepsilon_{\alpha}:=\frac{\alpha-2}{d+\alpha} .
$$

We notice that the competitor $h$ is non-negative in $B_{1}$, thus we only need to prove the inequality

$$
\widetilde{W}(h)-\Theta_{+}-(1-\varepsilon)\left(\widetilde{W}(z)-\Theta_{+}\right) \leq 0 .
$$

We divide the proof into three steps.

Step 1. Using the properties of $q_{\nu}$, we first reduce the inequality (3.2) to a comparison of the energy of $\tilde{\varphi}$ to the one of $\varphi$. Precisely, in Subsection 3.1, we prove the inequality

$$
\widetilde{W}(h)-\Theta_{+}-(1-\varepsilon)\left(\widetilde{W}(z)-\Theta_{+}\right) \leq W_{0}(\tilde{\varphi})-(1-\varepsilon) W_{0}(\varphi) .
$$


Step 2. In Subsection 3.2 we prove that we can choose $q_{\nu}$ in such a way that the function $\phi:=c-q_{\nu}$ does not contain modes of the first $d$ eigenvalues on the spherical cap $S_{\delta_{0}}$. Precisely, we prove the following claim. For every $\delta_{0}>0$ there exists $\delta>0$ such that

for every $c \in H_{0}^{1}\left(S_{\delta_{0}}\right)$ satisfying $\left\|c-q_{e_{d} / 2}\right\|_{L^{2}\left(\partial B_{1}\right)} \leq \delta$ there exists $\nu \in \mathbb{R}^{d}$ such that $u_{\lambda} \in H_{0}^{1}\left(S_{\delta_{0}}\right)$ and $\int_{S_{\delta_{0}}} c(\theta) \phi_{j}(\theta) d \mathcal{H}^{d-1}(\theta)=\int_{S_{\delta_{0}}} q_{\nu}(\theta) \phi_{j}(\theta) d \mathcal{H}^{d-1}(\theta)$, for every $j=1, \ldots, d$,

where $\phi_{1}, \ldots, \phi_{d}$ are the first $d$, orthonormal in $L^{2}\left(\partial B_{1}\right)$, eigenfunctions of the Laplace-Beltrami operator on $S_{\delta_{0}}$ with Dirichlet boundary conditions on $\partial S_{\delta_{0}}$.

Step 3. In Subsection 3.3 we use Lemma 2.1 and the choice of $\nu$ from Step 2 to prove the inequality

$$
W_{0}(\tilde{\varphi})-(1-\varepsilon) W_{0}(\varphi) \leq 0
$$

which together with (3.3) gives (3.2).

3.1. Decomposition of the energy. We prove (3.3) in the following lemma:

Lemma 3.1. Let $\alpha>2, \varepsilon_{\alpha}$ as in (3.1), $\nu=\left(\nu_{1}, \ldots, \nu_{d}\right) \in \mathbb{R}^{d}$ and $q_{\nu}(x)=(x \cdot \nu)_{+}^{2}$. Suppose that $\phi \in H^{1}\left(\partial B_{1}\right), \varphi(r, \theta)=r^{2} \phi(\theta)$ and $\tilde{\varphi}(r, \theta)=r^{\alpha} \phi(\theta)$. Then

$$
\left(\widetilde{W}\left(q_{\nu}+\tilde{\varphi}\right)-\Theta_{+}\right)-\left(1-\varepsilon_{\alpha}\right)\left(\widetilde{W}\left(q_{\nu}+\varphi\right)-\Theta_{+}\right) \leq W_{0}(\tilde{\varphi})-\left(1-\varepsilon_{\alpha}\right) W_{0}(\varphi) .
$$

Proof. Suppose, without loss of generality that, $q_{\nu}(x)=c_{0} q(x)$, where for the sake of simplicity we set $q:=q_{e_{d} / 2} \in \mathcal{K}_{+}$. Notice that for every $\psi \in H^{1}\left(B_{1}\right)$ we have

$$
\begin{aligned}
\widetilde{W}\left(c_{0} q+\psi\right)-\Theta_{+} & =c_{0}^{2} \int_{B_{1}}|\nabla q|^{2}-2 c_{0}^{2} \int_{\partial B_{1}} q^{2}+c_{0} \int_{B_{1}} q-\frac{1}{2} \int_{B_{1}} q \\
& \quad+2 c_{0}\left(\int_{B_{1}} \nabla q \cdot \nabla \psi-2 \int_{\partial B_{1}} q \psi\right)+\int_{B_{1}}|\nabla \psi|^{2}-2 \int_{\partial B_{1}} \psi^{2}+\int_{B_{1}} \psi \\
= & -\frac{\left(c_{0}-1\right)^{2}}{2} \int_{B_{1}} q+2 c_{0}\left(-\int_{B_{1}} \Delta q \psi+\int_{\partial B_{1}} \partial_{r} q \psi-2 \int_{\partial B_{1}} q \psi\right)+W_{0}(\psi)+\int_{B_{1}} \psi \\
= & -\left(c_{0}-1\right)^{2} \Theta_{+}+W_{0}(\psi)+\int_{B_{1}} \psi-c_{0} \int_{B_{1}^{+}} \psi,
\end{aligned}
$$

where we used that $\Theta_{+}=\frac{1}{2} \int_{B_{1}} q, \Delta q=\frac{1}{2} \chi_{B_{1}^{+}}$and $\partial_{r} q=2 q$. If $\psi=\tilde{\varphi}=r^{2} \phi$, then we have

$$
\int_{B_{1}} \tilde{\varphi}-c_{0} \int_{B_{1}^{+}} \tilde{\varphi}=\frac{1}{d+\alpha}\left(\int_{\partial B_{1}} \phi-c_{0} \int_{\partial B_{1}^{+}} \phi\right)=: \frac{1}{d+\alpha} \beta(\phi),
$$

and we can write the energy of $c_{0} q+\tilde{\varphi}$ in the form

$$
\widetilde{W}\left(c_{0} q+\tilde{\varphi}\right)-\Theta_{+}=-\left(c_{0}-1\right)^{2} \Theta_{+}+W_{0}(\tilde{\varphi})+\frac{1}{d+\alpha} \beta(\phi) .
$$

Applying the above estimate to $\varphi$ and $\tilde{\varphi}$ and thanks to the definition of $\varepsilon_{\alpha}$, we get

$$
\begin{aligned}
\widetilde{W}\left(c_{0} q+\tilde{\varphi}\right) & -\Theta_{+}-\left(1-\varepsilon_{\alpha}\right)\left(\widetilde{W}\left(c_{0} q+\varphi\right)-\Theta_{+}\right) \\
& =-\varepsilon_{\alpha}\left(c_{0}-1\right)^{2} \Theta_{+}+W_{0}(\tilde{\varphi})-\left(1-\varepsilon_{\alpha}\right) W_{0}(\varphi)+\left(\frac{1}{d+\alpha}-\frac{1-\varepsilon_{\alpha}}{d+2}\right) \beta(\phi) \\
& =-\varepsilon_{\alpha}\left(c_{0}-1\right)^{2} \Theta_{+}+W_{0}(\tilde{\varphi})-\left(1-\varepsilon_{\alpha}\right) W_{0}(\varphi),
\end{aligned}
$$

which concludes the proof of Lemma 3.1. 
3.2. Choice of $\nu$. In this section we prove the claim (3.4), which is a straightforward consequence of the following lemma.

Lemma 3.2. Given $\delta_{0}>0$, we denote by $S_{\delta_{0}}$ the set $\left\{x_{d}>-\delta_{0}\right\} \cap \partial B_{1}$ and by $\phi_{1}, \ldots, \phi_{d}$ the first $d$ eigenfunctions on the set $S_{\delta_{0}}$. Then the function

$$
F: \mathbb{R}^{d} \rightarrow \mathbb{R}^{d}, \quad F(\nu)=\left(\int_{S_{\delta_{0}}} q_{\nu} \phi_{1}, \ldots, \int_{S_{\delta_{0}}} q_{\nu} \phi_{d}\right)
$$

is a $C^{1}$ diffeomorphism in a neighbourhood $U \subset \mathbb{R}^{d}$ of $\frac{e_{d}}{2}$.

Proof of Lemma 3.2. We first notice that $F$ is a $C^{1}$ function in a neighborhood of $e_{d} / 2$, because the function $\mathbb{R}^{d} \times \mathbb{R}^{d} \ni(x, y) \mapsto(x \cdot y)_{+}^{2}$ is $C^{1}$. We now calculate the partial derivatives of $F=\left(F_{1}, \ldots, F_{d}\right)$ in $e_{d} / 2$. Using the fact that the first eigenfunction depends only on one varibale, $\phi_{1}=\phi_{1}\left(x_{d}\right)$, and that the higher eigenfunctions can be written in the form $\phi_{j}(x)=x_{j-1} \phi\left(x_{d}\right)$, for every $j=2, \ldots, d$ (see Subsection 2.5), we get that

$$
F_{1}(\nu)=\int_{\partial B_{1}} q_{\nu}(x) \phi_{1}\left(x_{d}\right) d x \quad \text { and } \quad F_{j}(\nu)=\int_{\partial B_{1}} q_{\nu}(x) x_{j-1} \phi\left(x_{d}\right) d x, \quad \forall j=2, \ldots, d .
$$

Setting $\partial B_{1}^{+}=\left\{x_{d}>0\right\} \cap \partial B_{1}$ we have

$$
\begin{gathered}
\frac{\partial F_{1}}{\partial \nu_{d}}\left(e_{d} / 2\right)=\int_{\partial B_{1}^{+}} x_{d}^{2} \phi_{1}\left(x_{d}\right) d x>0, \\
\frac{\partial F_{1}}{\partial \nu_{j}}\left(e_{d} / 2\right)=\int_{\partial B_{1}^{+}} x_{d} x_{j} \phi_{1}\left(x_{d}\right) d x=0, \forall j=1, \ldots, d-1 .
\end{gathered}
$$

where the positivity of the first term follows from the positivity of $\phi_{1}$, while the second term is zero since $x_{j}$ is odd. Moreover, for every $j=2, \ldots, d$ and $i=1, \ldots, d$, we have

$$
\frac{\partial F_{j}}{\partial \nu_{i}}\left(e_{d} / 2\right)=\int_{\partial B_{1}^{+}} x_{i} x_{d} x_{j-1} \phi\left(x_{d}\right) d x=\delta_{i(j-1)} \int_{\partial B_{1}^{+}} x_{i}^{2} x_{d} \phi\left(x_{d}\right) d x=\frac{\delta_{i(j-1)}}{d-1} \int_{\partial B_{1}^{+}}\left(1-x_{d}^{2}\right) x_{d} \phi\left(x_{d}\right) d x,
$$

where we used the fact that $x_{i}$ and $x_{j}$ are odd for the first equality, and $\int x_{j}^{2}=\frac{1}{d-1} \sum_{j=1}^{d-1} \int x_{j}^{2}=$ $\frac{1}{d-1} \int\left(1-x_{d}^{2}\right)$. By the positivity of $\phi$ and the fact that $\lim _{\delta_{0} \rightarrow 0}\left\|\phi-c_{2} x_{d}^{+}\right\|_{L^{2}\left(\partial B_{1}\right)}=0$, for dimensional constants $c_{1}$ and $c_{2}$ (which is due to the fact that on the half-sphere $\partial B_{1}^{+}$the eigenfunctions are of the form $\phi_{j}(x)=c_{2} x_{d} x_{j-1}$ for $\left.j=2, \ldots, d\right)$ we get that for $\delta_{0}$ small enough $D F\left(e_{d} / 2\right)$ is an invertible matrix and so, by the inverse function theorem there is a neighborhood of $e_{d} / 2$ on which $F$ is a $C^{1}$ diffeomorphism.

3.3. Homogeneity improvement of $\varphi$. We prove (3.5). Indeed, by the fact that the Fourier expansion of $\phi(\theta):=c(\theta)-q_{\nu}(\theta)$ does not contain the first $d$ modes $\phi_{1}, \ldots, \phi_{d}$ on the spherical cap $S_{\delta_{0}}$ (claim (3.4)), we obtain that the function $\phi$ can be expanded in Fourier series as

$$
\phi(\theta)=\sum_{j=d+1}^{\infty} c_{j} \phi_{j}(\theta) \quad \text { on the spherical cap } \quad S_{\delta_{0}}=\partial B_{1} \cap\left\{x_{d}>-\delta_{0}\right\} .
$$

Thus, by Lemma 2.1 we get

$$
W_{0}(\tilde{\varphi})-\left(1-\varepsilon_{\alpha}\right) W_{0}(\varphi)=\frac{\varepsilon_{\alpha}}{d+2 \alpha-2} \sum_{j=d+1}^{\infty}\left(-\lambda_{j}+\lambda_{\alpha}\right) c_{j}^{2},
$$

where $\lambda_{j}$ are the eigenvalue of the Dirichlet Laplacian on $S_{\delta_{0}}$ and $\lambda_{\alpha}=\alpha(\alpha+d-2)$. On the other hand, for $\delta_{0}>0$ small enough, we have that $\lambda_{j} \geq 3 d$, for $j \geq d+1$ (see Subsection 2.5), so that $-\lambda_{j}+\lambda_{\alpha} \leq 0$ whenever $\alpha>2$ and $\alpha(\alpha+d-2) \leq 3 d$. . Thus, choosing for instance

$$
\alpha=\frac{5}{2} \quad \text { and } \quad \varepsilon=\frac{\alpha-2}{d+\alpha}=\frac{1}{2 d+5},
$$

we conclude the proof of (3.5) and Theorem 2 , 


\section{The EPIPERIMETRIC INEQUALity For SINGUlar Points: PROOF of TheOREM 1}

We first notice that given any two-homogeneous function $z(r, \theta)=r^{2} c(\theta)$, we can decompose it in Fourier series on the sphere $\partial B_{1}$ as

$$
c(\theta)=\sum_{j=1}^{\infty} c_{j} \phi_{j}(\theta)=c_{1} \phi_{1}(\theta)+\sum_{\left\{j: \lambda_{j}=d-1\right\}} c_{j} \phi_{j}(\theta)+\sum_{\left\{j: \lambda_{j}=2 d\right\}} c_{j} \phi_{j}(\theta)+\sum_{\left\{j: \lambda_{j}>2 d\right\}} c_{j} \phi_{j}(\theta) .
$$

Therefore $z$ can be decomposed in a unique way as

$$
z=q_{\nu}+Q_{A}+\varphi
$$

where

(i) $\nu \in \mathbb{R}^{d}$ is such that $q_{\nu}(x)=(x \cdot \nu)_{+}^{2}$ contains in its Forurier expansion precisely the sum

$$
\sum_{\left\{j: \lambda_{j}=d-1\right\}} c_{j} \phi_{j}(\theta)
$$

(ii) $A$ is a symmetric matrix depending on the coefficients $c_{j}$, corresponding to the eigenvalues $\lambda_{j}=0, d-1,2 d$, and $Q_{A}(x)=x \cdot A x$

(iii) $\varphi$ is a two-homogeneous function, in polar coordinates $\varphi(r, \theta)=r^{2} \phi(\theta)$, containing only higher modes on $\partial B_{1}$, that is the trace $\phi$ can be written in the form

$$
\phi(\theta)=\sum_{\left\{j: \lambda_{j}>2 d\right\}} c_{j} \phi_{j}(\theta)
$$

where $\left\{\phi_{j}\right\}_{j \in \mathbb{N}}$ are the eigenfunctions of the spherical laplacian as in (2.4) with $S=\partial B_{1}$.

Notice that, in the above representation $A$ might not be positive definite. Let $B$ be a symmetric positive definite matrix and $Q_{B}(x)=x \cdot B x$. Then, $z$ can be rewritten as

$$
z=q_{\nu}+Q_{B}+\left(Q_{A}-Q_{B}\right)+\varphi .
$$

We then replace the 2-homogeneous function $\psi:=\left(Q_{A}-Q_{B}\right)+\varphi$ by an $\alpha$-homogeneous function $\tilde{\psi}$ with the same boundary values as $\psi$. We will choose $\alpha>2$ such that

$$
\varepsilon_{\alpha}:=\frac{\alpha-2}{d+\alpha}=\varepsilon\left(C_{4}\left\|\nabla_{\theta} \phi\right\|_{L^{2}\left(\partial B_{1}\right)}^{2}\right)^{\gamma},
$$

where $C_{4}$ is the dimensional constant from the inequality (4.7) and $\gamma$ is the constant from (1.2). Subsequently we will choose $\varepsilon$ to be small enough, but yet depending only on the dimension.

Finally, the competitor $h$ is given by

$$
h=q_{\nu}+Q_{B}+\tilde{\psi}
$$

Since $\inf \{\psi, 0\} \leq \inf \{\tilde{\psi}, 0\}$ and $q_{\nu}+Q_{B} \geq 0$, by the choice of $B, h$ is non-negative in $B_{1}$ and so we only need to prove the inequality

$$
W(h) \leq W(z)-\varepsilon(W(z)-\Theta)^{1+\gamma} .
$$

The proof of Theorem 1 will be carried out in four steps.

Step 1. In Subsection 4.1 we set $c_{0}=4 \sum_{j=1}^{d} \nu_{j}$ and $b=4 \operatorname{tr} B$ and we prove the identity

$$
\begin{aligned}
W(h) & -\Theta-\left(1-\varepsilon_{\alpha}\right)(W(z)-\Theta) \\
& =-\frac{\varepsilon_{\alpha}}{2}\left(\left(1-b-c_{0}\right)^{2}+(1-b)^{2}\right) \Theta+W_{0}(\tilde{\psi})-\left(1-\varepsilon_{\alpha}\right) W_{0}(\psi) .
\end{aligned}
$$

Step 2. We now choose $Q_{B}$. If $A$ is positive definite $\left(Q_{A} \geq 0\right)$, then we choose $B=A$. If $Q_{A}$ changes sign, then up to a change of coordinates we can assume that there exist $a_{j} \geq 0$ for every $j=1, \ldots, d$ such that

$$
Q_{A}(x)=-\sum_{j=1}^{k} a_{j} x_{j}^{2}+\sum_{j=k+1}^{d} a_{j} x_{j}^{2}, \quad a_{d} \geq \frac{1}{4 d}>\sum_{j=1}^{k} a_{j},
$$


where the last inequality being due to the fact that $Q_{A}$ is $L^{2}\left(\partial B_{1}\right)$-close to the set of admissible blow-ups $\mathcal{K}$. We set

$$
Q_{B}(x):=\sum_{j=k+1}^{d} a_{j} x_{j}^{2}-\left(\sum_{j=1}^{k} a_{j}\right) x_{d}^{2} \geq 0
$$

where the last inequality, that is the positive definiteness of $B$, depends on $a_{d} \geq \frac{1}{4 d}>\sum_{j=1}^{k} a_{j}$. In Subsection 4.2 we then prove that there exists a dimensional constant $C_{2}>0$ such that

$$
W_{0}(\tilde{\psi})-\left(1-\varepsilon_{\alpha}\right) W_{0}(\psi) \leq \varepsilon_{\alpha}^{2} C_{2} \sum_{j=1}^{k} a_{j}^{2}-\frac{\varepsilon_{\alpha}}{2}\left\|\nabla_{\theta} \phi\right\|_{L^{2}\left(\partial B_{1}\right)}^{2} .
$$

Step 3. In Subsection 4.3 we prove that there are dimensional constants $C_{3}>0$ and $\gamma \in[0,1)$ such that

$$
\sum_{j=1}^{k} a_{j}^{2} \leq C_{3}\left\|\nabla_{\theta} \phi\right\|_{L^{2}\left(\partial B_{1}\right)}^{2(1-\gamma)} .
$$

In the supplementary Subsection 4.5 we show that this estimate can be improved in several ways: - in dimension two (4.6) holds with $\gamma=0$;

- the dimensional constants $\gamma$ can be replaced by a (smaller) constant $\gamma_{k}$, this time depending on $d$ and $k$. In the two extremal cases $k=0$ and $k=d-1$ the constant is zero.

Step 4. In Subsection 4.4 we prove that there is a dimensional constant $C_{4}$, such that

$$
W(z)-\Theta \leq C_{4}\left\|\nabla_{\theta} \phi\right\|_{L^{2}\left(\partial B_{1}\right)}^{2}
$$

Conclusion of the proof. The proof of Theorem 1 now follows directly by (4.3), (4.5), (4.6) and (4.7). Indeed, by (4.5) and (4.6) we get that

$$
\begin{aligned}
W_{0}(\tilde{\psi})-\left(1-\varepsilon_{\alpha}\right) W_{0}(\psi) & \leq \varepsilon_{\alpha}^{2} C_{2} \sum_{j=1}^{k} a_{j}^{2}-\frac{\varepsilon_{\alpha}}{2}\left\|\nabla_{\theta} \phi\right\|_{L^{2}\left(\partial B_{1}\right)}^{2} \\
& \leq \varepsilon_{\alpha}^{2} C_{2} C_{3}\left\|\nabla_{\theta} \phi\right\|_{L^{2}\left(\partial B_{1}\right)}^{2(1-\gamma)}-\frac{\varepsilon_{\alpha}}{2}\left\|\nabla_{\theta} \phi\right\|_{L^{2}\left(\partial B_{1}\right)}^{2} .
\end{aligned}
$$

By the definition of $\varepsilon_{\alpha}$ and (4.7) we get

$$
\begin{aligned}
W_{0}(\tilde{\psi})-\left(1-\varepsilon_{\alpha}\right) W_{0}(\psi) & \leq \varepsilon^{2} C_{4}^{2 \gamma}\left\|\nabla_{\theta} \phi\right\|_{L^{2}\left(\partial B_{1}\right)}^{4 \gamma} C_{2} C_{3}\left\|\nabla_{\theta} \phi\right\|_{L^{2}\left(\partial B_{1}\right)}^{2(1-\gamma)}-\frac{\varepsilon}{2} C_{4}^{\gamma}\left\|\nabla_{\theta} \phi\right\|_{L^{2}\left(\partial B_{1}\right)}^{2 \gamma}\left\|\nabla_{\theta} \phi\right\|_{L^{2}\left(\partial B_{1}\right)}^{2} \\
& =\varepsilon C_{4}^{\gamma}\left(\varepsilon C_{2} C_{3} C_{4}^{\gamma}-\frac{1}{2}\right)\left\|\nabla_{\theta} \phi\right\|_{L^{2}\left(\partial B_{1}\right)}^{2+2 \gamma},
\end{aligned}
$$

which is negative for $\varepsilon$ small enough (but yet, $\varepsilon$ depends only on the dimension). Finally, by (4.3), the definition of $\varepsilon_{\alpha}$ and (4.7) we obtain

$$
\begin{aligned}
W(h)-\Theta & \leq\left(1-\varepsilon_{\alpha}\right)(W(z)-\Theta)=\left(1-\varepsilon C_{4}^{\gamma}\left\|\nabla_{\theta} \phi\right\|_{L^{2}\left(\partial B_{1}\right)}^{2 \gamma}\right)(W(z)-\Theta) \\
& \leq\left(1-\varepsilon(W(z)-\Theta)^{\gamma}\right)(W(z)-\Theta),
\end{aligned}
$$

which is precisely (1.2). We now proceed with the proof of (4.3), (4.5), (4.6) and (4.7).

4.1. Decomposition of the energy. We prove the following lemma, which implies easily (4.3).

Lemma 4.1. Let $\alpha>2$ and $\varepsilon_{\alpha}=\frac{\alpha-2}{d+\alpha}$; let $0 \neq \nu=\left(\nu_{1}, \ldots, \nu_{d}\right) \in \mathbb{R}^{d}, q_{\nu}(x)=(x \cdot \nu)_{+}^{2}$, $c_{0}=4 \sum_{j=1}^{d} \nu_{j}^{2}$; let $B$ be a symmetric matrix with and $b=4 \operatorname{tr} B \neq 0$ and $Q_{B}(x)=x \cdot B x$. Suppose that $\phi \in H^{1}\left(\partial B_{1}\right), \varphi(r, \theta)=r^{2} \phi(\theta)$ and $\tilde{\varphi}(r, \theta)=r^{\alpha} \phi(\theta)$. Then

$$
\begin{aligned}
\widetilde{W}\left(q_{\nu}+Q_{B}+\tilde{\varphi}\right) & -\Theta-\left(1-\varepsilon_{\alpha}\right)\left(\widetilde{W}\left(q_{\nu}+Q_{B}+\varphi\right)-\Theta\right) \\
& =-\frac{\varepsilon_{\alpha}}{2}\left(\left(1-b-c_{0}\right)^{2}+(1-b)^{2}\right) \Theta+W_{0}(\tilde{\varphi})-\left(1-\varepsilon_{\alpha}\right) W_{0}(\varphi) .
\end{aligned}
$$


Proof. We $Q=\frac{1}{b} Q_{B}$. Thus, we have $Q \in \mathcal{K}$. In particular, $\Delta Q=1 / 2$ in $B_{1}$ and $W(Q)=\Theta$. We notice that for every function $\eta \in H^{1}\left(B_{1}\right)$, we have

$$
\begin{aligned}
\widetilde{W}(b Q+\eta)-\Theta & =b^{2} W_{0}(Q)+W_{0}(\eta)+2 b\left(\int_{B_{1}} \nabla Q \cdot \nabla \eta-2 \int_{\partial B_{1}} Q \eta\right)+b \int_{B_{1}} Q+\int_{B_{1}} \eta-\Theta \\
& =b^{2} W_{0}(Q)+W_{0}(\eta)-2 b \int_{B_{1}} \eta \Delta Q+b \int_{B_{1}} Q+\int_{B_{1}} \eta-\Theta,
\end{aligned}
$$

which gives

$$
\widetilde{W}(b Q+\eta)-\Theta=-(1-b)^{2} \Theta+W_{0}(\eta)+(1-b) \int_{B_{1}} \eta .
$$

We set $q=\frac{1}{c_{0}} q_{\nu}$. Thus $q \in \mathcal{K}_{+}$and $W(q)=\Theta_{+}=\frac{1}{2} \int_{B_{1}} q$. Setting $\eta=c_{0} q+\psi$ in (4.8) we obtain

$$
\begin{aligned}
\widetilde{W}\left(b Q+c_{0} q+\psi\right)-\Theta= & -(1-b)^{2} \Theta+W_{0}\left(c_{0} q+\psi\right)+(1-b) \int_{B_{1}}\left(c_{0} q+\psi\right) \\
= & -(1-b)^{2} \Theta+c_{0}^{2} W_{0}(q)+W_{0}(\psi) \\
& +2 c_{0}\left(\int_{B_{1}} \nabla q \cdot \nabla \psi-2 \int_{\partial B_{1}} q \psi\right)+(1-b) \int_{B_{1}}\left(c_{0} q+\psi\right) \\
= & -(1-b)^{2} \Theta-c_{0}^{2} \frac{\Theta}{2}+W_{0}(\psi)-2 c_{0} \int_{B_{1}} \psi \Delta q+(1-b) c_{0} \Theta+(1-b) \int_{B_{1}} \psi \\
= & -\frac{\left(1-b-c_{0}\right)^{2}+(1-b)^{2}}{2} \Theta+W_{0}(\psi)+\beta(\psi),
\end{aligned}
$$

where in the last line we set

$$
\beta(\psi):=(1-b) \int_{B_{1}} \psi-c_{0} \int_{B_{1}^{+}} \psi
$$

Taking $\tilde{\psi}$ to be the $\alpha$-homogeneous extension of $\psi$, we get that $\beta(\tilde{\psi})-\left(1-\varepsilon_{\alpha}\right) \beta(\psi)=0$, which concludes the proof of the lemma.

4.2. Homogeneity improvement of $\psi$. In this subsection we prove the inequality (4.5).

We first notice that if $Q_{A}$ is non-negative, then we can choose $Q_{B}=Q_{A}$ and $\sum_{j=1}^{k} a_{j}^{2}=0$. Thus, (4.5) follows directly by Lemma 2.1 and the fact that for the eigenvalues on the sphere $\lambda_{j}>2 d$ implies $\lambda_{j} \geq 3(d+1)$.

In the rest of this subsection, we assume that $Q_{A}$ changes sign and $Q_{B}$ is given by (4.4). In particular, $Q_{A}-Q_{B}$ is a homogeneous polynomial of second degree with $\Delta Q_{A}-Q_{B}=0$, so it is an element of the eigenspace $E_{2 d}$, corresponding to the eigenvalue $2 d$. We choose $\phi_{2} \in E_{2 d}$ and $c_{2} \in \mathbb{R}$ such that

$$
Q_{A}-Q_{B}=c_{2} \phi_{2}, \quad \text { where } \quad \int_{\partial B_{1}} \phi_{2}^{2}(\theta) d \mathcal{H}^{d-1}(\theta)=1 .
$$

Thus, on $\partial B_{1}$ we can write $\psi$ as

$$
\psi(\theta)=c_{2} \phi_{2}(\theta)+\phi(\theta)=c_{2} \phi_{2}(\theta)+\sum_{\left\{j: \lambda_{j}>2 d\right\}} c_{j} \phi_{j}(\theta) .
$$

Applying Lemma 2.1 we have

$$
\begin{aligned}
W_{0}(\tilde{\psi})-\left(1-\varepsilon_{\alpha}\right) W_{0}(\psi) & =\frac{\varepsilon_{\alpha}}{d+2 \alpha-2}\left((\alpha-2)(d+\alpha) c_{2}^{2}+\sum_{\left\{j: \lambda_{j}>2 d\right\}}\left(-\lambda_{j}+\lambda_{\alpha}\right) c_{j}^{2}\right) \\
& =\frac{\varepsilon_{\alpha}^{2}(d+\alpha)^{2}}{(d+2 \alpha-2)} c_{2}^{2}+\frac{\varepsilon_{\alpha}}{d+2} \sum_{\left\{j: \lambda_{j}>2 d\right\}}\left(-\lambda_{j}+\lambda_{\alpha}\right) c_{j}^{2}
\end{aligned}
$$


Choosing the constant $\varepsilon$ small enough, the equation (4.2) implies that $2<\alpha \leq \frac{5}{2}$; by the fact that $\lambda_{j}>2 d \Rightarrow \lambda_{j} \geq 3(d+1)$ (see Subsection 2.5 ) we have

$$
\lambda_{j}-\lambda_{\alpha} \geq 3(d+1)-\frac{5}{2}\left(d+\frac{1}{2}\right)>\frac{d+2}{2}, \quad \text { whenever } \quad \lambda_{j}>2 d .
$$

Hence, the right-hand side in the previous equality can be estimated by

$$
\begin{aligned}
W_{0}(\tilde{\psi})-\left(1-\varepsilon_{\alpha}\right) W_{0}(\psi) & \leq \frac{\varepsilon_{\alpha}^{2}(d+3)^{2}}{(d+2)} c_{2}^{2}-\frac{\varepsilon_{\alpha}}{2} \sum_{\left\{j: \lambda_{j}>2 d\right\}} c_{j}^{2} \\
& =\frac{\varepsilon_{\alpha}^{2}(d+3)^{2}}{(d+2)} \int_{\partial B_{1}}\left(Q_{A}-Q_{B}\right)^{2}-\frac{\varepsilon_{\alpha}}{2} \int_{\partial B_{1}} \phi^{2} .
\end{aligned}
$$

It order to estimate the first term in the right-hand side, we notice that $Q_{A}-Q_{B}=\left(\sum_{j=1}^{k} a_{j}\right) x_{d}^{2}-$ $\sum_{j=1}^{k} a_{j} x_{j}^{2}$, hence its $L^{2}$-norm is a degree 2 homogeneous polynomial in $\left(a_{1}, \ldots, a_{k}\right)$ (with coefficients depending only on $d$ ). Hence there exists a dimensional constant $C_{d}$ such that

$$
\int_{\partial B_{1}}\left(Q_{A}-Q_{B}\right)^{2} \leq C_{d} \sum_{j=1}^{k} a_{j}^{2}
$$

Together with (4.10), this gives (4.5).

4.3. The higher modes control $\sum_{j=1}^{k} a_{j}^{2}$. In this section we prove the inequality (4.6) from Step 3. Since the trace $c(\theta)$ is positive on $\partial B_{1}$ and can be written as

$$
c(\theta)=\left(-\sum_{j=1}^{k} a_{j} \theta_{j}^{2}+\sum_{j=k+1}^{d} a_{j} \theta_{j}^{2}\right)+q_{\nu}(\theta)+\phi(\theta) \geq 0,
$$

we get that

$$
\phi(\theta) \geq\left(\sum_{j=1}^{k} a_{j} \theta_{j}^{2}-\sum_{j=k+1}^{d} a_{j} \theta_{j}^{2}\right)_{+} \geq\left(a_{1} \theta_{1}^{2}-\sum_{j=2}^{d} \theta_{j}^{2}\right)_{+}
$$

on the half-sphere $\partial B_{1} \cap\left\{q_{\nu}=0\right\}$. Thus, we get

$$
\phi(\theta) \geq \frac{a_{1}}{4} \theta_{1}^{2} \quad \text { on the set } \quad U_{a_{1}} \cap\left\{q_{\nu}=0\right\}, \quad \text { where } \quad U_{a_{1}}=\left\{\theta \in \partial B_{1}: a_{1} \theta_{1}^{2}>2 \sum_{j=2}^{d} \theta_{j}^{2}\right\} .
$$

Notice that that for $a_{1}$ small enough we have

$$
\frac{1}{2}(d-1) \omega_{d-1}{\sqrt{a_{1}}}^{d-1} \leq \mathcal{H}^{d-1}\left(U_{a_{1}}\right) \leq 2(d-1) \omega_{d-1}{\sqrt{a_{1}}}^{d-1} .
$$

Thus, we obtain

$$
\int_{\partial B_{1}} \phi^{2} \geq C_{d} a_{1}^{2}{\sqrt{a_{1}}}^{d-1}=C_{d} a_{1}^{(d+3) / 2},
$$

for a dimensional constant $C_{d}>0$. Without loss of generality we can suppose that

$$
a_{1}^{2} \geq \frac{1}{k} \sum_{j=1}^{k} a_{j}^{2} \geq \frac{1}{d} \sum_{j=1}^{k} a_{j}^{2}
$$

and so, we get

$$
\int_{\partial B_{1}}\left|\nabla_{\theta} \phi\right|^{2} \geq 2 d \int_{\partial B_{1}} \phi^{2} \geq 2 d C_{d} a_{1}^{(d+3) / 2} \geq C_{d}\left(\sum_{j=1}^{k} a_{j}^{2}\right)^{(d+3) / 4},
$$

which gives (4.6) with $\gamma=\frac{d-1}{d+3}$ and a dimensional constant $C_{3}$. 
4.4. The higher modes control $W(z)-\Theta$. In this section we prove the inequality (4.7) from the final Step 4. Using the decomposition $z=q_{\nu}+Q_{A}+\psi$ and the identity (4.9) we get, with $\psi=r^{2} \phi$ and using $\int_{\partial B_{1}} \phi=0$ since it contains only high modes,

$$
\begin{aligned}
W(z)-\Theta & =-\frac{\left(1-b-c_{0}\right)^{2}+(1-b)^{2}}{2} \Theta+\frac{1}{d+2} \int_{\partial B_{1}}\left(\left|\nabla_{\theta} \phi\right|^{2}-2 d \phi^{2}\right)-\frac{c_{0}}{d+2} \int_{\partial B_{1}^{+}} \phi \\
& \leq-\frac{c_{0}^{2}}{4} \Theta+\frac{1}{d+2} \int_{\partial B_{1}}\left(\left|\nabla_{\theta} \phi\right|^{2}-2 d \phi^{2}\right)-\frac{c_{0}}{d+2} \int_{\partial B_{1}^{+}} \phi,
\end{aligned}
$$

where the last inequality follows by the fact that

$$
\left(1-b-c_{0}\right)^{2}+(1-b)^{2} \geq \frac{c_{0}^{2}}{2}, \quad \text { for every } \quad b, c_{0} \in \mathbb{R} .
$$

By the Cauchy-Schwarz inequality, we have

$$
\begin{aligned}
-\frac{c_{0}}{d+2} \int_{\partial B_{1}^{+}} \phi & \leq \frac{c_{0}^{2}}{4} \Theta+\left(\frac{1}{(d+2) \Theta} \int_{\partial B_{1}^{+}} \phi\right)^{2} \\
& \leq \frac{c_{0}^{2}}{4} \Theta+\frac{\left|\partial B_{1}\right|}{(d+2) \Theta} \int_{\partial B_{1}} \phi^{2}=\frac{c_{0}^{2}}{4} \Theta+8 d^{2} \int_{\partial B_{1}} \phi^{2} .
\end{aligned}
$$

Thus, we get

$$
\begin{aligned}
W(z)-\Theta & \leq \frac{1}{d+2} \int_{\partial B_{1}}\left(\left|\nabla_{\theta} \phi\right|^{2}-2 d \phi^{2}\right)+8 d^{2} \int_{\partial B_{1}} \phi^{2} \\
& \leq \frac{1}{d+2} \int_{\partial B_{1}}\left|\nabla_{\theta} \phi\right|^{2}+8 d^{2} \int_{\partial B_{1}} \phi^{2} \leq\left(\frac{1}{d+2}+4 d\right) \int_{\partial B_{1}}\left|\nabla_{\theta} \phi\right|^{2},
\end{aligned}
$$

where the last inequality is due to the fact that $\phi$ contains only modes $\phi_{j}$ corresponding to eigenvalues $\lambda_{j}>2 d$. This gives (4.7) where the constant $C_{4}$ can be choosen as $C_{4}=1+4 d$.

4.5. Improvement of the decay rate. This subsection is dedicated to the improvement of the inequality (4.6). The main result, contained in the following lemma, is more general and holds in any dimension.

Lemma 4.2. Suppose that $0 \leq k<d$ and

$$
Q_{A}(x)=-\sum_{j=1}^{k} a_{j} x_{j}^{2}+\sum_{j=k+1}^{d} a_{j} x_{j}^{2}, \quad \text { where }\left\{\begin{array}{l}
0<a_{j} \quad \text { for every } j=1, \ldots, k, \\
0 \leq a_{j} \leq 1 \quad \text { for every } j=k+1, \ldots, d
\end{array}\right.
$$

Let $\phi \in H^{1}\left(\partial B_{1}\right)$ be of zero mean, that is $\int_{\partial B_{1}} \phi(\theta) d \mathcal{H}^{d-1}(\theta)=0$, and such that

$$
\phi \geq Q_{A} \text { on the half-sphere }\left\{\xi \in \partial B_{1}: \xi \cdot \nu>0\right\},
$$

determined by some unit vector $\nu \in \partial B_{1}$.

Then, there are dimensional constants $C>0$ and $\delta>0$ such that if $\sum_{j=1}^{k} a_{j}^{2} \leq \delta$, then

$$
\sum_{j=1}^{k} a_{j}^{2} \leq C_{d}\left\|\nabla_{\theta} \varphi\right\|_{L^{2}\left(\partial B_{1}\right)}^{2\left(1-\gamma_{k}\right)}
$$

where

$$
\gamma_{k}=\left\{\begin{array}{l}
0, \quad \text { if } k=0 \\
\frac{d-k}{d-k+4}, \quad \text { for every } k=1, \ldots, d-2 \\
0, \quad \text { if } \quad k=d-1
\end{array}\right.
$$


Remark 4.3. The above lemma is to be applied to the traces $c$ of the solutions $u$ of an obstacle problem, which can be written in the form $c(\theta)=Q_{A}(\theta)+q_{\nu}(\theta)+\phi(\theta)$. We notice that, although one might think that $k$ corresponds precisely to point of the $k$-th stratum, we do not know a way to deduce the precise form of $Q_{A}$ just from looking at the blow-up limits of $u$. This means that even if the blow up $Q_{B}$ is $\operatorname{such}$ that $\operatorname{dim}(\operatorname{ker} B)=k$, we still cannot infer anything on the structure of $B$. It follows that this result cannot be applied to improve the regularity of the singular sets of $\partial\{u>0\}$, except in dimension two, where $\gamma_{0}=\gamma_{1}=0$. This corresponds to the assumption of Weiss on the projection of $c$ on the set of admissible blow-ups $\mathcal{K}$, which again finds application only in dimension two.

Proof of Lemma 4.2. If $k=0$, then the inequality is trivial and so, we can suppose that $k \geq 1$.

Suppose that $1 \leq k<d-1$. Without loss of generality we can suppose $a_{1}^{2} \geq \frac{1}{k} \sum_{i=1}^{k} a_{i}^{2}$.

Setting $X^{\prime}=\left(x_{1}, \ldots, x_{k}\right), X^{\prime \prime}=\left(x_{k+1}, \ldots, x_{d}\right)$ and $\|\cdot\|=\|\cdot\|_{L^{2}\left(\partial B_{1}\right)}$, we have

$$
\begin{aligned}
\left\|\left(\sum_{i=1}^{k} a_{i} x_{i}^{2}-\sum_{i=k+1}^{d} a_{i} x_{i}^{2}\right)_{+}\right\| & \geq\left\|\left(a_{1} x_{1}^{2}-\sum_{i=k+1}^{d} a_{i} x_{i}^{2}\right)_{+}\right\| \geq\left\|\left(a_{1} x_{1}^{2}-\left|X^{\prime \prime}\right|^{2}\right)_{+}\right\| \\
& =\frac{1}{k} \sum_{j=1}^{k}\left\|\left(a_{1} x_{j}^{2}-\left|X^{\prime \prime}\right|^{2}\right)_{+}\right\|
\end{aligned}
$$

We now notice that

$$
a_{1}\left|X^{\prime}\right|^{2}-\left|X^{\prime \prime}\right|^{2} \geq \frac{a_{1}}{2}\left|X^{\prime}\right|^{2} \quad \text { on the set } \quad U_{a_{1}}=\left\{X=\left(X^{\prime}, X^{\prime \prime}\right) \in \partial B_{1}: \frac{a_{1}}{2}\left|X^{\prime}\right|^{2} \geq\left|X^{\prime \prime}\right|^{2}\right\},
$$

and for $a_{1}$ small enough we get

$$
\frac{1}{2} k \omega_{k}{\sqrt{a_{1}}}^{d-k} \leq \mathcal{H}^{d-1}\left(U_{a_{1}}\right) \leq 2 k \omega_{k}{\sqrt{a_{1}}}^{d-k} .
$$

In particular,

$$
\left\|\inf \left\{Q_{A}, 0\right\}\right\|_{L^{2}\left(\partial B_{1}\right)}^{2} \geq\left\|\left(a_{1}\left|X^{\prime}\right|^{2}-\left|X^{\prime \prime}\right|^{2}\right)_{+}\right\|_{L^{2}\left(\partial B_{1}\right)}^{2} \geq C_{d} a_{1}^{\frac{d-k+4}{2}} \geq C_{d}\left(\sum_{i=1}^{k} a_{i}^{2}\right)^{\frac{d-k+4}{4}} .
$$

Now, since $Q_{A}$ is even and $\|\phi\|_{L^{2}\left(\partial B_{1}\right)}^{2} \leq \frac{1}{d-1}\left\|\nabla_{\theta} \phi\right\|_{L^{2}\left(\partial B_{1}\right)}^{2}$, we obtain the claimed inequality

$$
\sum_{i=1}^{k} a_{i}^{2} \leq C_{d}\left\|\nabla_{\theta} \phi\right\|_{L^{2}\left(\partial B_{1}\right)}^{\frac{8}{d-k+4}}
$$

Suppose that $k=d-1$. We argue by contradiction. Suppose that there are a sequence of functions $\phi_{n}: \partial B_{1} \rightarrow \mathbb{R}$ of zero mean and vectors $\nu_{n} \in \partial B_{1}$ and $\left(a_{n}^{1}, \ldots, a_{n}^{k}\right)$ such that

$$
\begin{gathered}
\phi_{n}(\theta) \geq a_{n}^{1} \theta_{1}^{2} \quad \text { on the set } \quad\left\{\theta \in \partial B_{1}: \theta_{d}=0, \theta \cdot \nu_{n}>0\right\}, \\
a_{n}^{1} \geq\left(\frac{1}{k} \sum_{j=1}^{k}\left|a_{n}^{j}\right|^{2}\right)^{1 / 2} \quad \text { and } \quad \sum_{j=1}^{k}\left|a_{n}^{j}\right|^{2} \geq n\left\|\nabla \phi_{n}\right\|_{L^{2}\left(\partial B_{1}\right)}^{2} .
\end{gathered}
$$

Thus, the sequence of functions $\psi_{n}:=\phi_{n} / a_{n}^{1}$ is such that $\lim _{n \rightarrow \infty}\left\|\nabla \psi_{n}\right\|_{L^{2}\left(\partial B_{1}\right)}^{2}=0$ and

$$
\psi_{n}(\theta) \geq \theta_{1}^{2} \quad \text { on the set } \quad\left\{\theta \in \partial B_{1}: \theta_{d}=0, \theta \cdot \nu_{n}>0\right\},
$$

which is in contradiction with the trace inequality

$$
\int_{\left\{\theta_{d}=0\right\} \cap \partial B_{1}} \psi_{n}^{2} d \mathcal{H}^{d-2} \leq C \int_{\partial B_{1}}\left(\left|\nabla \psi_{n}\right|^{2}+\psi_{n}^{2}\right) d \mathcal{H}^{d-1}
$$


4.6. On the sharpness of the non-homogeneous estimate in Theorem 1. We conclude this section with an example, which shows that in dimension higher than three one cannot estimate the distance to the cone $\mathcal{K}$ by just using the energy of the higher modes $\phi$ to the power one. Indeed, such an estimate would be in contradiction with inequality (4.12) below. In particular, Example 1 shows that for general traces in higher dimension our method cannot be improved.

Example 1. Consider the non-negative trace $c: \partial B_{1} \rightarrow \mathbb{R}^{+}$given by

$$
c(\theta)=\left(\frac{1}{4(d-1)} \sum_{j=1}^{d-1} \theta_{j}^{2}-\varepsilon \theta_{d}^{2}\right)_{+} .
$$

Notice that, since $c$ is even its Fourier expansion on the sphere $\partial B_{1}$ does not contain linear terms. As in the proof of Theorem 1, the trace $c$ can be uniquely decomposed as $c(\theta)=Q(\theta)+\phi(\theta)$, where $Q$ is a homogeneous polynomial of second degree and $\phi$ contains only higher modes, that is

$$
\phi(\theta)=\sum_{\left\{j: \lambda_{j}>2 d\right\}} c_{j} \phi_{j}(\theta) .
$$

We claim that

$$
\left\|\nabla_{\theta} \phi\right\|_{L^{2}\left(\partial B_{1}\right)}^{\frac{4}{d+1}} \lesssim \operatorname{dist}_{L^{2}\left(\partial B_{1}\right)}(Q, \mathcal{K}) .
$$

In order to prove (4.12) we set

$$
P(\theta)=\frac{1}{4(d-1)} \sum_{j=1}^{d-1} \theta_{j}^{2}-\varepsilon \theta_{d}^{2} \quad \text { and } \quad R(\theta)=\left(\varepsilon \theta_{d}^{2}-\frac{1}{4(d-1)} \sum_{j=1}^{d-1} \theta_{j}^{2}\right)_{+},
$$

and we notice that $c(\theta)=P(\theta)-R(\theta)$. It is easy to check that the term $R$ has the following asymptotic behavior when the parameter $\varepsilon$ is small:

$$
\begin{gathered}
\|R\|_{L^{\infty}\left(\partial B_{1}\right)}=\varepsilon, \quad \mathcal{H}^{d-1}(\{R>0\}) \sim \varepsilon^{\frac{d-1}{2}}, \quad\left\|\nabla_{\theta} R\right\|_{L^{\infty}\left(\partial B_{1}\right)} \sim \sqrt{\varepsilon}, \\
\|R\|_{L^{2}\left(\partial B_{1}\right)} \sim \varepsilon^{\frac{d+3}{4}} \quad \text { and } \quad\left\|\nabla_{\theta} R\right\|_{L^{2}\left(\partial B_{1}\right)} \sim \varepsilon^{\frac{d+1}{4}} .
\end{gathered}
$$

The function $R$ can be decomposed as

$$
R(\theta)=\frac{c_{0}}{\sqrt{\mathcal{H}^{d-1}\left(\partial B_{1}\right)}}+c_{2} \phi_{2}(\theta)-\phi(\theta),
$$

where

- $c_{0} \in \mathbb{R}$ corresponds to the first (constant) mode of the Fourier expansion of $R$ on $\partial B_{1}$ and can be estimated in terms of $\varepsilon$ as

$$
c_{0}=\frac{1}{\sqrt{\mathcal{H}^{d-1}\left(\partial B_{1}\right)}} \int_{\partial B_{1}} R d \mathcal{H}^{d-1} \leq\|R\|_{L^{2}\left(\partial B_{1}\right)} \lesssim \varepsilon^{\frac{d+3}{4}} ;
$$

- $\phi_{2}(\theta)$ is an eigenfunction of the Laplacian on the sphere corresponding to the eigenvalue $2 d$ and $\left\|\phi_{2}\right\|_{L^{2}\left(\partial B_{1}\right)}=1$ and the constant $c_{2} \in \mathbb{R}$ can be estimated as

$$
\left|c_{2}\right| \leq\left|\int_{\partial B_{1}} R \phi_{2}\right| \leq\|R\|_{L^{2}\left(\partial B_{1}\right)} \lesssim \varepsilon^{\frac{d+3}{4}} ;
$$

- the function $\phi$ is precisely the one from the decomposition of c, contains only higher modes and satisfies the following estimate:

$$
\left\|\nabla_{\theta} \phi\right\|_{L^{2}\left(\partial B_{1}\right)} \leq\left\|\nabla_{\theta} R\right\|_{L^{2}\left(\partial B_{1}\right)}+\left|c_{2}\right|\left\|\nabla_{\theta} \phi_{2}\right\|_{L^{2}\left(\partial B_{1}\right)} \lesssim \varepsilon^{\frac{d+1}{4}}+\varepsilon^{\frac{d+3}{4}} 2 d \lesssim \varepsilon^{\frac{d+1}{4}} .
$$

On the other hand, the $L^{2}\left(\partial B_{1}\right)$ distance from $Q=P-c_{0}-c_{2} \phi_{2}$ to the cone $\mathcal{K}$ of nonnegative homogeneous polynomials of second degree has the behavior

$$
\operatorname{dist}_{L^{2}\left(\partial B_{1}\right)}\left(P-c_{0}-c_{2} \phi_{2}, \mathcal{K}\right) \sim \operatorname{dist}_{L^{2}\left(\partial B_{1}\right)}(P, \mathcal{K}) \sim \varepsilon
$$


Thus, we finally get the claimed inequality (4.12)

$$
\left\|\nabla_{\theta} \phi\right\|_{L^{2}\left(\partial B_{1}\right)}^{\frac{4}{d+1}} \lesssim \varepsilon \sim \operatorname{dist}_{L^{2}\left(\partial B_{1}\right)}\left(P-c_{0}-c_{2} \phi_{2}, \mathcal{K}\right) .
$$

\section{UNIQUENESS OF BLOW-UP AND REGULARITY OF FREE BOUNDARY}

In this Section we prove Theorems 3 and 4 , focusing on the statement 2 of each result. We show in detail how the logarithmic estimates follow from the "modified" epiperimetric inequality of Theorem 1 and we prefer to skip the analogous estimates on the Hölder continuity at regular points, since this is the main improvement of the present paper and since the proof of the latter is a simpler version of the estimates below and it is already contained in [14, Theorem 4 and 5].

Proposition 5.1. Let $\Omega \subset \mathbb{R}^{d}$ be an open set and $u \in H^{1}(\Omega)$ a minimizer of $\mathcal{E}$. Then for every compact set $K \Subset \Omega$, there is a constant $C:=C(d, K, \Omega)>0$ such that for every free boundary point $x_{0} \in \operatorname{Sing}(u) \cap K$, the following decay holds

$$
\left\|u_{x_{0}, t}-u_{x_{0}, s}\right\|_{L^{1}\left(\partial B_{1}\right)} \leq C(-\log (t))^{-\frac{1-\gamma}{2 \gamma}} \quad \text { for all } \quad 0<s<t<\operatorname{dist}(K, \partial \Omega) .
$$

Proof. Step 1 (closeness of the blow ups for a given point $x_{0}$ ). Let $x_{0} \in K$ and let $r_{0} \in$ $(0, \operatorname{dist}(K, \partial \Omega)]$ be such that the epiperimetric inequality of Theorem 1 can be applied to the rescaling $u_{x_{0}, r}$ for every $r \leq r_{0}$. We claim that

$$
\left\|u_{x_{0}, t}-u_{x_{0}, s}\right\|_{L^{1}\left(\partial B_{1}\right)} \leq C\left(-\log \left(t / r_{0}\right)\right)^{-\frac{1-\gamma}{2 \gamma}} \quad \text { for all } \quad 0<s<t<r_{0} .
$$

We assume $x_{0}=0$ without loss of generality and

$$
e(r)=W(u, r)-\Theta_{u}(0) .
$$

By the monotonicity formula (2.1) and the epiperimetric inequality of Theorem 11, there exists a radius $r_{0}>0$ such that for every $r \leq r_{0}$

$$
\frac{d}{d r} e(r) \geq \frac{d+2}{r}\left(W\left(c_{r}\right)-\Theta_{u}(0)-e(r)\right)+f(r) \geq \frac{c}{r} e(r)^{1+\gamma}+2 f(r)
$$

where $\gamma \in(0,1)$ is a dimensional constant and

$$
f(r):=\frac{1}{r} \int_{\partial B_{1}}\left|x \cdot \nabla u_{r}-2 u_{r}\right|^{2} d \mathcal{H}^{1} .
$$

We obtain that

$$
\frac{d}{d r}\left(\frac{-1}{\gamma e(r)^{\gamma}}-c \log r\right)=\frac{1}{e(r)^{1+\gamma}} \frac{d}{d r} e(r)-\frac{c}{r} \geq \frac{1}{e(r)^{1+\gamma}} f(r) \geq 0
$$

and this in turn implies that $-e(r)^{-\gamma}-c \gamma \log r$ is an increasing function of $r$, namely that $e(r)$ decays as

$$
e(r) \leq\left(e\left(r_{0}\right)^{-\gamma}+c \gamma \log r_{0}-c \gamma \log r\right)^{\frac{-1}{\gamma}} \leq\left(-c \gamma \log \left(r / r_{0}\right)\right)^{\frac{-1}{\gamma}}
$$

For any $0<s<t<r_{0}$ we estimate the $L^{1}$ distance between the blow ups at scale $s$ and $t$ through the Cauchy-Schwarz inequality and the monotonicity formula (2.1)

$$
\begin{aligned}
\int_{\partial B_{1}}\left|u_{t}-u_{s}\right| d \mathcal{H}^{n-1} & \leq \int_{\partial B_{1}} \int_{s}^{t} \frac{1}{r}\left|x \cdot \nabla u_{r}-2 u_{r}\right| d r d \mathcal{H}^{n-1} \\
& \leq\left(n \omega_{n}\right)^{1 / 2} \int_{s}^{t} \frac{1}{r}\left(\frac{1}{r} \int_{\partial B_{1}}\left|x \cdot \nabla u_{r}-2 u_{r}\right|^{2} d \mathcal{H}^{n-1}\right)^{1 / 2} d r \\
& \leq\left(\frac{n \omega_{n}}{2}\right)^{1 / 2} \int_{s}^{t} \frac{1}{r}\left(e^{\prime}(r)\right)^{1 / 2} d r \\
& \leq\left(\frac{n \omega_{n}}{2}\right)^{1 / 2}(\log (t)-\log (s))^{1 / 2}(e(t)-e(s))^{1 / 2}
\end{aligned}
$$


Let $0<s^{2}<t^{2}<r_{0}$ such that $s / r_{0} \in\left[2^{-2^{i+1}}, 2^{-2^{i}}\right), t / r_{0} \in\left[2^{-2^{j+1}}, 2^{-2^{j}}\right)$ for some $j \leq i$ and applying the previous estimate(5.4) to the exponentially dyadic decomposition, we obtain

$$
\begin{aligned}
\int_{\partial B_{1}}\left|u_{t}-u_{s}\right| d \mathcal{H}^{n-1} & \leq \int_{\partial B_{1}}\left|u_{t}-u_{2^{-2^{j+1}} r_{0}}\right| d \mathcal{H}^{n-1} \\
& +\int_{\partial B_{1}}\left|u_{2^{-2^{i}} r_{0}}-u_{s}\right| d \mathcal{H}^{n-1}+\sum_{k=j+1}^{i-1} \int_{\partial B_{1}}\left|u_{2^{-2^{k+1}} r_{0}}-u_{2^{-2^{k}} r_{0}}\right| d \mathcal{H}^{n-1} \\
& \leq C \sum_{k=j}^{i}\left(\log \left(2^{-2^{k}}\right)-\log \left(2^{-2^{k+1}}\right)\right)^{1 / 2}\left(e\left(2^{-2^{k}}\right)-e\left(2^{-2^{k+1}}\right)\right)^{1 / 2} \\
& \leq C \sum_{k=j}^{i} 2^{k / 2} e\left(2^{-2^{k}}\right)^{1 / 2} \leq C \sum_{k=j}^{i} 2^{(1-1 / \gamma) k / 2} \\
& \leq C 2^{(1-1 / \gamma) j / 2} \leq C\left(-\log \left(t / r_{0}\right)\right)^{\frac{\gamma-1}{2 \gamma}}
\end{aligned}
$$

where $C$ is a dimensional constant that may vary from line to line.

Step 2 (uniform smallness of monotonic quantity for $\left.x_{0} \in \operatorname{Sing}(u) \cap K\right)$. We claim that for every $\varepsilon>0$ there exists $r_{0}>0$ such that

$$
e\left(u_{x, r}\right) \leq \varepsilon \quad \text { for every } x \in \operatorname{Sing}(u) \cap K, r \leq r_{0} .
$$

Assume by contradiction that there exists a sequence $x_{k} \rightarrow x_{0}$ and $r_{k} \rightarrow 0$ such that $\varepsilon<$ $e\left(u_{x_{k}, r_{k}}\right)$ for any $k \in \mathbb{N}$. By the monotonicity of $W$, for any $\rho>0$ and $k$ large enough

$$
\varepsilon<W\left(u, x_{k}, r_{k}\right)-\Theta_{u}(0) \leq W\left(u, x_{k}, \rho\right)-W\left(u, x_{0}, \rho\right)+W\left(u, x_{0}, \rho\right)-\Theta_{u}(0) .
$$

In turn, the right-hand side can be made arbitrarily small by choosing first $\rho$ sufficiently small (to make the difference of the last two terms small) and then $k$ sufficiently large.

Step 3 (uniform scale for the application of the epiperimetric inequality at $\left.x_{0} \in \operatorname{Sing}(u) \cap K\right)$. We claim that for every $\varepsilon>0$ there exists $r_{0}>0$ such that

$$
\operatorname{dist}_{L^{2}}\left(u_{x, r}, K\right) \leq \varepsilon \quad \text { for every } x \in \operatorname{Sing}(u) \cap K, r \leq r_{0} .
$$

(notice that this statement holds also if in place of the $L^{2}$-distance we consider the $H^{1}$-distance).

Assume by contradiction that there exists $\varepsilon>0$ a sequence $x_{k} \rightarrow x_{0}$ and $r_{k} \rightarrow 0$ such that

$$
\varepsilon<\operatorname{dist}_{L^{2}}\left(u_{x_{k}, r_{k}}, K\right) \quad \text { for any } k \in \mathbb{N} \text {. }
$$

Since the sequence $\left\{u_{x_{k}, r_{k}}\right\}_{k \in \mathbb{N}}$ is uniformly bounded in $H^{2, \infty}$, it converges strongly in $H^{1}$ up to a (not relabelled) subsequence to $u_{0}$. Moreover, thanks to Step 2, the limit $u_{0}$ must satisfy $W\left(u_{0}, x_{0}, 1\right)=\Theta_{u}(0)$, so that it belongs to $K$. This contradicts (5.7).

Step 4 (conclusion). We can now conclude the proof of the Proposition.

We observe that for every $r_{0}>0$ and $t \leq r_{0}^{2}$, we have $\log \left(t / r_{0}\right) \leq 2 \log t$. From Step 1 and 3 , we deduce that there exists $r_{0}>0$ such that for all $0<s<t<r_{0}^{2}, x_{0} \in \operatorname{Sing}(u) \cap K$

$$
\left\|u_{x_{0}, t}-u_{x_{0}, s}\right\|_{L^{1}\left(\partial B_{1}\right)} \leq C(-\log (t))^{-\frac{1-\gamma}{2 \gamma}} .
$$

From (5.5) we have

$$
\left\|u_{x_{0}, t}-u_{x_{0}, r_{0}^{2}}\right\|_{L^{1}\left(\partial B_{1}\right)} \leq C\left(-\log \left(r_{0}\right)\right)^{1 / 2} e(\operatorname{dist}(K, \partial \Omega))^{1 / 2}
$$

and the right hand side is estimated by $C\left(-\log \left(r_{0}\right)\right)^{-\frac{1-\gamma}{2 \gamma}}$ for a constant $C$ depending only on $d, r_{0}, e(\operatorname{dist}(K, \partial \Omega)), \operatorname{dist}(K, \partial \Omega)$.

As a consequence of the previous proposition we can prove the uniqueness of the blow up Theorem 3, with a logarithmic rate of convergence of the blow up sequence at each point of the singular set (and uniform in any compact set inside the domain). 
Proof of Theorem 3. We notice that

$$
\left|Q_{x_{1}}-Q_{x_{2}}\right| \leq c(n) \int_{\partial B_{1}}\left|Q_{x_{1}}(x)-Q_{x_{2}}(x)\right| d \mathcal{H}^{n-1}(x)
$$

By the triangular inequality

$$
\left\|Q_{x_{1}}-Q_{x_{2}}\right\|_{L^{1}\left(\partial B_{1}\right)} \leq\left\|u_{x_{1}, r}-Q_{x_{1}}\right\|_{L^{1}\left(\partial B_{1}\right)}+\left\|u_{x_{1}, r}-u_{x_{2}, r}\right\|_{L^{1}\left(\partial B_{1}\right)}+\left\|u_{x_{2}, r}-Q_{x_{2}}\right\|_{L^{1}\left(\partial B_{1}\right)}
$$

Recalling that $u \in C^{1,1}$ and that $\nabla u\left(x_{1}\right)=0$, we estimate the term in the middle with

$$
\begin{aligned}
\left\|u_{x_{1}, r}-u_{x_{2}, r}\right\|_{L^{1}\left(\partial B_{1}\right)} & \leq \int_{\partial B_{1}} \int_{0}^{1} \frac{\left|\nabla u\left(x_{1}+r x+t\left(x_{2}-x_{1}\right)\right)\right|\left|x_{2}-x_{1}\right|}{r^{2}} d t d \mathcal{H}(x) \\
& \leq C\|u\|_{C^{1,1}\left(B_{r}\left(x_{0}\right)\right)} \frac{\left(r+\left|x_{2}-x_{1}\right|\right)\left|x_{2}-x_{1}\right|}{r^{2}}
\end{aligned}
$$

We choose $r=\left|x_{1}-x_{2}\right|\left(-\log \left|x_{1}-x_{2}\right|\right)^{-\frac{1-\gamma}{2 \gamma}}$ and we assume that $r_{0}$ satisfies the inequality $\left|r_{0}\right|\left(-\log \left|r_{0}\right|\right)^{-\frac{1-\gamma}{2 \gamma}} \leq \operatorname{dist}(K, \partial \Omega)$. By Theorem 3 we see that

$$
\begin{aligned}
\left\|u_{x_{1}, r}-Q_{x_{1}}\right\|_{L^{1}\left(\partial B_{1}\right)}+\left\|u_{x_{2}, r}-Q_{x_{2}}\right\|_{L^{1}\left(\partial B_{1}\right)} & \leq C(-\log (r))^{-\frac{1-\gamma}{2 \gamma}} \\
& =C\left(-\log \left|x_{1}-x_{2}\right|-\frac{1-\gamma}{2 \gamma} \log \left(\log \left|x_{1}-x_{2}\right|\right)\right)^{-\frac{1-\gamma}{2 \gamma}}
\end{aligned}
$$

Noticing that the inequality $a-\frac{1-\gamma}{2 \gamma} \log a \geq a / 2$ holds for $a$ greater than a given $a_{0}>0$ (depending only on $\gamma$ and therefore on $d$ ), we apply this inequality to $a=-\log \left|x_{1}-x_{2}\right|$ to get

$$
\left\|u_{x_{1}, r}-Q_{x_{1}}\right\|_{L^{1}\left(\partial B_{1}\right)}+\left\|u_{x_{2}, r}-Q_{x_{2}}\right\|_{L^{1}\left(\partial B_{1}\right)} \leq C\left(-\log \left|x_{1}-x_{2}\right|\right)^{-\frac{1-\gamma}{2 \gamma}} .
$$

Putting together the previous inequalities, we find (1.8).

5.1. Proof of Theorem 5. We notice that if $u \in H^{1}(\Omega)$ is a minimizer of $\mathcal{E}_{q}$ or $\mathcal{A}_{q}$, then it is locally $W^{2, \infty}$ by the results of 10 and moreover it is an almost-minimizer of the functional $\mathcal{E}$ with a constant $C$ depending only on $\|q\|_{C^{0, \gamma}(\Omega)}, c_{q}$ and $\|u\|_{W_{l o c}^{2, \infty}}$.

We say that $u \in H^{1}(\Omega)$ is an almost minimizer of $\mathcal{E}$ if there exists a constant $C>0$ such that for every ball $B_{r}\left(x_{0}\right) \subset \Omega$ and for every $v \in H^{1}\left(B_{r}\left(x_{0}\right)\right)$ which agrees with $u$ on $\partial B_{r}\left(x_{0}\right)$

$$
\int_{B_{r}\left(x_{0}\right)}\left[|\nabla u|^{2}+q\left(x_{0}\right) \max \{u, 0\}\right] d x \leq\left(1+C r^{\gamma}\right) \int_{B_{r}\left(x_{0}\right)}\left[|\nabla v|^{2}+q\left(x_{0}\right) \max \{v, 0\}\right] d x .
$$

In the following we show that the statement of Theorem 5, in particular the logarithmic estimate, holds true also if we drop the assumption that $u \in W_{l o c}^{2, \infty}(\Omega)$ is a minimizer of $\mathcal{E}_{q}$ or $\mathcal{A}_{q}$ and we only assume the almost minimality.

The main modifications with respect to the arguments of Section 5 appear in Proposition 5.1 and we outline them below.

Up to a rescaling, we may assume that $q\left(x_{0}\right)=1$. Applying the epiperimetric inequality of Theorem 10 to $u_{r, x_{0}}$, we find that (5.2) has to be modified for almost monotonicity (5.10) to get

$$
\frac{d}{d r} e(r) \geq \frac{n+2}{r}\left(W\left(c_{r}\right)-\Theta_{u}(0)-e(r)\right)+f(r) \geq \frac{c_{0}}{r} e(r)^{1+\gamma}-\frac{c_{1}}{r^{1-\alpha}}+2 f(r)
$$

(where $e(r):=W\left(u_{r}, 1\right)-\Theta_{u}(0)$ and the notation is the same as in Section 5) for some constants $c_{0}, c_{1}>0$.

We define now $\tilde{e}(r)=e(r)+2 \alpha^{-1} c_{1} r^{\alpha}$ and we notice that from the previous inequality and since $a^{1+\gamma}+b^{1+\gamma} \geq 2^{-\gamma}(a+b)^{1+\gamma}$ for any $a, b \geq 0$

$$
\tilde{e}^{\prime}(r) \geq \frac{c_{0}}{r} e(r)^{1+\gamma}+\frac{c_{1}}{r^{1-\alpha}}+2 f(r) \geq \frac{c_{0}}{r}\left[e(r)+c_{1} r^{\frac{\alpha}{1+\gamma}}\right]^{1+\gamma}+2 f(r)
$$

For $r$ sufficiently small, the previous inequality implies that

$$
\tilde{e}^{\prime}(r) \geq \frac{c_{0}}{r} \tilde{e}(r)^{1+\gamma}+2 f(r)
$$


From the previous inequality, we see that $\tilde{e}(r)$ satisfies the same inequality that $e(r)$ solved in (5.2). Hence, with the same argument as in (5.3), we see that $\tilde{e}$ satisfies the same estimate as $e$ in (5.4)

$$
e(r)+2 \alpha^{-1} c_{1} r^{\alpha}=\tilde{e}(r) \leq\left(-c \gamma \log \left(r / r_{0}\right)\right)^{\frac{-1}{\gamma}} .
$$

This inequality implies that, up to a constant, also $e(r)$ satisfies a logarithmic estimate and we can carry out the rest of the proof of Proposition 5.1 and of Theorems 3 and 4 .

\section{REFERENCES}

[1] David Adams and Leon Simon. Rates of asymptotic convergence near isolated singularities of geometric extrema. Indiana Univ. Math. J., 37(2):225-254, 1988.

[2] L. A. Caffarelli. The obstacle problem revisited. J. Fourier Anal. Appl., 4(4-5):383-402, 1998.

[3] L. A. Caffarelli and N. M. Rivière. Smoothness and analyticity of free boundries in variational inequalities. Ann. Scuola Norm. Sup. Pisa Cl. Sci., 3 (2):289-310, 1976.

[4] Luis A. Caffarelli. The regularity of free boundaries in higher dimensions. Acta Math., 139(3-4):155-184, 1977.

[5] M. Colombo, L. Spolaor, and B. Velichkov. Direct epiperimetric inequalities for the thin obstacle problem. In preparation, 2017.

[6] M. Focardi, M. S. Gelli, and E. Spadaro. Monotonicity formulas for obstacle problems with Lipschitz coefficients. Calc. Var. Partial Differential Equations, 54(2):1547-1573, 2015.

[7] M. Focardi, F. Geraci, and E. Spadaro. The classical obstacle problem for nonlinear variational energies. Nonlinear Anal., 154:71-87, 2017.

[8] Matteo Focardi and Emanuele Spadaro. An epiperimetric inequality for the thin obstacle problem. Adv. Differential Equations, 21(1-2):153-200, 2016.

[9] Nicola Garofalo, Arshak Petrosyan, and Mariana Smit Vega Garcia. An epiperimetric inequality approach to the regularity of the free boundary in the Signorini problem with variable coefficients. J. Math. Pures Appl. (9), 105(6):745-787, 2016.

[10] Claus Gerhardt. Global $C^{1,1}$-regularity for solutions of quasilinear variational inequalities. Arch. Rational Mech. Anal., 89(1):83-92, 1985.

[11] Regis Monneau. A brief overview on the obstacle problem. In European Congress of Mathematics, Vol. II (Barcelona, 2000), volume 202 of Progr. Math., pages 303-312. Birkhäuser, Basel, 2001.

[12] E. R. Reifenberg. An epiperimetric inequality related to the analyticity of minimal surfaces. Ann. of Math. (2), 80:1-14, 1964.

[13] L. Spolaor and B. Velichkov. Regularity of free boundary in the one-phase problem i: dimension 2. Preprint, 2016.

[14] Georg S. Weiss. A homogeneity improvement approach to the obstacle problem. Invent. Math., 138(1):23-50, 1999.

[15] Georg Sebastian Weiss. Partial regularity for a minimum problem with free boundary. J. Geom. Anal., $9(2): 317-326,1999$.

[16] Brian White. Tangent cones to two-dimensional area-minimizing integral currents are unique. Duke Math. J., 50(1):143-160, 1983.

Maria Colombo:

Institute for TheoreticAl Studies, ETH ZÜRICH,

Clausiusstrasse 47, CH-8092 Zürich, Switzerland

E-mail address: maria.colombo@eth-its.ethz.ch

LuCA SPOLAOR:

Massachusetts Institute of Technology (MIT),

77 Massachusetts Avenue, Cambridge MA 02139, USA

E-mail address: 1spolaor@mit.edu

BOZHIDAR VELICHKOV:

Laboratoire Jean Kuntzmann (LJK), Université Grenoble Alpes

BÂtiment IMAG, 700 Avenue Centrale, 38401 Saint-Martin-D'Hères

E-mail address: bozhidar.velichkov@univ-grenoble-alpes.fr 